\title{
Periods of abelian varieties
}

\author{
J. S. Milne
}

ABSTRACT
We prove various characterizations of the period torsor of abelian varieties.

Contents

Introduction

1 Preliminaries

2 Periods of abelian varieties with complex multiplication

\section{Introduction}

For an abelian variety $A$ over $\mathbb{Q}, H^{1}(A(\mathbb{C}), \mathbb{C})$ has two $\mathbb{Q}$-structures, that provided by singular cohomology $H^{1}(A(\mathbb{C}), \mathbb{Q})$ and that provided by de Rham cohomology $\mathbb{H}^{1}\left(A_{\text {Zar }}, \Omega_{A / k}^{\bullet}\right)$. The periods of $A$ are the coefficients of the transition matrix from a $\mathbb{Q}$-basis for one structure to a $\mathbb{Q}$-basis for the other. It is known [Del82a] that Hodge classes on $A$ impose algebraic relations on the periods, and it is conjectured that these are the only such relations. Thus, there appears to be no hope of obtaining an explicit description of the periods themselves, but one may still hope to characterize some of the objects attached to them. The singular and de Rham cohomologies define fibre functors on the category of motives based on the abelian varieties over $\mathbb{Q}$, and the difference of these functors is measured by the period torsor $P^{\mathrm{AV}}$. In this paper, we obtain various characterizations of $P^{\mathrm{AV}}$. Beyond its intrinsic interest, the period torsor controls the rationality of automorphic vector bundles, and therefore of holomorphic automorphic forms (see [Mil88] and [Mil90, § III 4]).

The first problem one runs into is that $P^{\mathrm{AV}}$ is a torsor for an affine group scheme $G$ over $\mathbb{Q}$ for the flat (more specifically, the FPQC) topology. Such torsors are classified by the flat cohomology group $H^{1}(\mathbb{Q}, G)$ rather than a more familiar Galois cohomology group. For an algebraic quotient $G_{n}$ of $G$, the two cohomology groups coincide. In $\S 1$ we prove that the canonical map

$$
H^{1}(\mathbb{Q}, G) \rightarrow \lim _{\longleftarrow} H^{1}\left(\mathbb{Q}, G_{n}\right)
$$

is surjective, and that the fibre of the map containing the class of a $G$-torsor $P$ is

$$
\lim ^{1}\left(P \wedge^{G} G_{n}\right)(\mathbb{Q})
$$

(nonabelian higher inverse limit). The limits are over the set of algebraic quotients $G_{n}$ of $G$. We show, moreover, that the full group $H^{1}(\mathbb{Q}, G)$ can be interpreted as a Galois cohomology group, but it is Galois cohomology defined using cochains that are continuous relative to the inverse limit topology on $\lim _{n} G_{n}\left(\mathbb{Q}^{\text {al }}\right)$ (discrete topology on each $G_{n}\left(\mathbb{Q}^{\text {al }}\right)$ ). It is important to note that these results depend crucially on the fact that the algebraic quotients of $G$ form a countable set; I do not

Received 3 March 2003, accepted in final form 4 June 2003.

2000 Mathematics Subject Classification 11J95 (primary), 11G10, 14K15, 18 G50 (secondary).

Keywords: abelian varieties, periods, flat cohomology.

Partially supported by the National Science Foundation.

This journal is (C) Foundation Compositio Mathematica 2004. 


\section{J. S. Milne}

even know how to define a nonabelian higher inverse limit except for countable coefficient sets. The remainder of $\S 1$ reviews results, more or less known, concerning nonabelian higher inverse limits and the classification of morphisms of torsors.

In $\S 2$, we take up the problem of characterizing $P^{\mathrm{CM}}$, the period torsor for the category of motives based on abelian varieties of (potential) CM-type over $\mathbb{Q}$. Since, as Deligne has pointed out, the period torsor $P^{\text {Art }}$ attached to the subcategory of Artin motives can be explicitly described, it is natural rather to consider the 'relative' problem of characterizing the morphism $P^{\mathrm{CM}} \rightarrow P^{\text {Art }}$ of torsors. Objects of this type are classified by the flat cohomology group $H^{1}\left(\mathbb{Q},{ }_{f} S\right)$ where ${ }_{f} S$ is a certain twist of the Serre group. As the Serre group is commutative, the main result of $\S 1$ simplifies to an exact sequence

$$
0 \rightarrow \lim ^{1}{ }_{f} S_{n}(\mathbb{Q}) \rightarrow H^{1}\left(\mathbb{Q},{ }_{f} S\right) \rightarrow \lim _{\longleftarrow} H^{1}\left(\mathbb{Q},{ }_{f} S_{n}\right) \rightarrow 0 .
$$

Blasius (unpublished) showed that $\lim ^{1} H^{1}\left(\mathbb{Q},{ }_{f} S_{n}\right)$ satisfies a Hasse principle, and [Win90] showed that $\lim _{\longleftarrow} H^{1}\left(\mathbb{Q},{ }_{f} S_{n}\right)=0$. If $\lim _{f}{ }_{f} S_{n} \overleftarrow{(\mathbb{Q})}$ were also zero, then $P^{\mathrm{CM}} \rightarrow P^{\text {Art }}$ would be unique up to an isomorphism inducing the identity on $P^{\text {Art }}$. Alas, it is not zero; in fact, we show that $\lim _{f}^{1} S_{n}(\mathbb{Q})$ is uncountable. Our proof of this uses an old theorem of Scholz and Reichardt on the embedding problem for Galois groups of number fields. It would be interesting to have more information on $\lim _{f}^{1} S_{n}(\mathbb{Q})$.

In $\S 3$, we take up the problem of characterizing $P^{\mathrm{AV}}$. Again, it is more natural to consider the relative problem of characterizing $P^{\mathrm{AV}} \rightarrow P^{\mathrm{CM}}$. Among other results, we prove that the isomorphism class of $P^{\mathrm{AV}} \rightarrow P^{\mathrm{CM}}$ is uniquely determined by its classes over $\mathbb{Q}_{l}(l=2,3,5, \ldots, \infty)$, about which a great deal is known.

Blasius and Borovoi [BB99] have studied the problem of characterizing $P^{\mathcal{H}}$ where $\mathcal{H} \varsubsetneqq \mathrm{AV}$ is the category of motives based on a certain class of abelian varieties over $\mathbb{Q}$ whose Mumford-Tate groups have simply connected derived group. Their main theorem [BB99, 1.5] states that the isomorphism class of $P^{\mathcal{H}} \rightarrow P^{\mathrm{CM}}$ is determined by the Galois cohomology class of $P^{\mathcal{H}}$ over $\mathbb{R}$. Unfortunately, their proof of this is inadequate for two reasons. First, they make the (false!) assumption that the flat cohomology groups coincide with the inverse limit Galois cohomology groups; this amounts to setting all the $\lim ^{1}$ equal to zero. Second, they misidentify the cohomology class that must be proved trivial for their theorem to hold. ${ }^{1}$ It seems unlikely that the statement of their theorem is correct (see Remark 3.26 below), but by combining Corollary 1.14 of this paper with their arguments, one obtains Theorem 3.25: the isomorphism class of $P^{\mathcal{H}} \rightarrow P^{\mathrm{CM}}$ is uniquely determined by its isomorphism class over $\mathbb{R}$. This observation began my work on this paper.

\section{Notations and conventions}

'Variety' means geometrically reduced scheme of finite type over a field. Semisimple algebraic groups are connected and 'simple' for an algebraic group means 'noncommutative and having no proper closed connected normal subgroup $\neq 1$ '. The identity component of a group scheme $G$ over a field is denoted by $G^{\circ}$. For a connected (pro)reductive group $G$ over a field, $Z G$ is the centre of $G, G^{\text {ad }}$ is the adjoint group $G / Z G$ of $G, G^{\text {der }}$ is the derived group of $G$, and $G^{\text {ab }}$ is the largest commutative quotient $G / G^{\text {der }}$ of $G$. The universal covering of a semisimple group $G$ is denoted $\tilde{G} \rightarrow G$.

The algebraic closure of $\mathbb{Q}$ in $\mathbb{C}$ is denoted by $\mathbb{Q}^{\text {al }}$, and (except in $\S 1$ ) $\Gamma=\operatorname{Gal}\left(\mathbb{Q}^{\text {al }} / \mathbb{Q}\right)$. We set $\operatorname{Gal}(\mathbb{C} / \mathbb{R})=\{1, \iota\}$. A CM-field is any field $E$ algebraic over $\mathbb{Q}$ admitting a nontrivial involution $\iota_{E}$ such that $\iota \circ \rho=\rho \circ \iota_{E}$ for all $\rho: E \rightarrow \mathbb{C}$.

\footnotetext{
${ }^{1}$ With their notations, in order to prove their theorem, they would have to show in $\S 5.1$ of their paper that the class of $\mathcal{P} \rightarrow \mathcal{P}^{\mathrm{CM}}$ in $H^{1}\left(\mathbb{Q},\left(\mathcal{G}_{\mathrm{DR}}^{\circ}\right)^{\text {der }}\right)$ is trivial (see Proposition 1.10 below). Instead, they prove only the weaker statement that the image of the class in $H^{1}\left(\mathbb{Q}, \mathcal{G}_{\mathrm{DR}}^{\circ}\right)$ is trivial, which, in fact, is all their hypotheses imply, even when one ignores the $\lim ^{1}$ terms.
} 


\section{PERIODS OF ABELIAN VARIETIES}

All categories of motives will be defined using absolute Hodge classes as the correspondences (see [Del82a] and [DM82, §6]).

We sometimes use $[x]$ to denote an equivalence or isomorphism class containing $x$. The notation $X \approx Y$ means that $X$ and $Y$ are isomorphic, and $X \cong Y$ means that $X$ and $Y$ are canonically isomorphic (or that a particular isomorphism is given).

\section{Preliminaries}

\section{Inverse limits}

For an inverse system of groups indexed by $(\mathbb{N}, \leqslant)$,

$$
\left(A_{n}, u_{n}\right)_{n \in \mathbb{N}}=\left(A_{0} \leftarrow \cdots \leftarrow A_{n-1} \stackrel{u_{n}}{\leftarrow} A_{n} \leftarrow \cdots\right),
$$

define $\lim ^{1} A_{n}$ to be the set of orbits for the left action of the group $\prod_{n} A_{n}$ on the set $\prod_{n} A_{n}$,

$$
\begin{array}{cccc}
\prod_{n} A_{n} & \times \quad \prod_{n} A_{n} & \rightarrow & \prod_{n} A_{n} \\
\left(\ldots, a_{n}, \ldots\right) & & \left(\ldots, x_{n}, \ldots\right) & \mapsto \quad\left(\ldots, a_{n} \cdot x_{n} \cdot u_{n+1}\left(a_{n+1}\right)^{-1}, \ldots\right) .
\end{array}
$$

This is a set, pointed by the orbit of $\underline{1}=(1,1, \ldots)$. Note that

$$
\lim _{\longleftarrow} A_{n}=\left\{a \in \prod A_{n} \mid a \cdot \underline{1}=\underline{1}\right\} .
$$

Let $\left(A_{n}\right)_{n \in \mathbb{N}} \rightarrow\left(B_{n}\right)_{n \in \mathbb{N}}$ be an inverse system of injective homomorphisms. From

$$
0 \rightarrow\left(A_{n}\right)_{n \in \mathbb{N}} \rightarrow\left(B_{n}\right)_{n \in \mathbb{N}} \rightarrow\left(B_{n} / A_{n}\right)_{n \in \mathbb{N}} \rightarrow 0
$$

we obtain an exact sequence

$$
1 \rightarrow \lim _{\longleftarrow} A_{n} \rightarrow \underset{\lim }{\longleftarrow} B_{n} \rightarrow \underset{\lim }{\longleftarrow}\left(B_{n} / A_{n}\right) \rightarrow \lim ^{1} A_{n} \rightarrow \lim ^{1} B_{n}
$$

of groups and pointed sets. Exactness at $\lim _{(}\left(B_{n} / A_{n}\right)$ means that the fibres of $\lim _{(}\left(B_{n} / A_{n}\right) \rightarrow \lim ^{1} A_{n}$ are the orbits for the natural action of $\longleftarrow \underset{\lim }{\longleftarrow} B_{n}$ on $\lim _{\longleftarrow}\left(B_{n} / A_{n}\right)$. When each $\overleftarrow{A_{n}}$ is normal in $B_{n}$, so that $C_{n}={ }_{\mathrm{df}} B_{n} / A_{n}$ is a group, (1) can be extended to an exact sequence

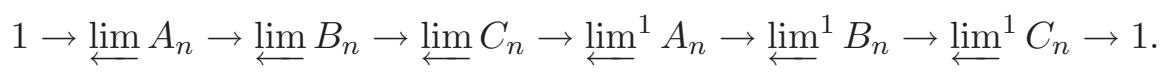

Exactness at $\lim ^{1} A_{n}$ means that the fibres of $\lim ^{1} A_{n} \rightarrow \lim ^{1} B_{n}$ are the orbits for the natural action of $\lim _{\longleftarrow} C_{n}$ on $\lim ^{1} A_{n}$.

Recall that an inverse system $\left(X_{n}\right)_{n \in \mathbb{N}}$ of sets (or groups) is said to satisfy the condition $(M L)$ if, for each $m$, the decreasing chain in $X_{m}$ of the images of the $X_{n}$ for $n \geqslant m$ is eventually constant.

Proposition 1.1. Let $\left(A_{n}, u_{n}\right)_{n \in \mathbb{N}}$ be an inverse system of groups.

a) If $\left(A_{n}, u_{n}\right)$ satisfies $(M L)$, then $\lim ^{1} A_{n}=0$.

b) If the $A_{n}$ are countable and $\left(A_{n}, u_{n}\right)_{n \in \mathbb{N}}$ fails $(M L)$, then $\varliminf^{1} A_{n}$ is uncountable.

Proof. a) The action ${ }^{2}$

$$
\left(\ldots, a_{n}, \ldots\right) \cdot\left(\ldots, x_{n}, \ldots\right)=\left(\ldots, a_{n} \cdot x_{n} \cdot\left(u a_{n+1}\right)^{-1}, \ldots\right)
$$

of the group $G_{N+1}={ }_{\mathrm{df}} \prod_{0 \leqslant n \leqslant N+1} A_{n}$ on the set $S_{N}={ }_{\mathrm{df}} \prod_{0 \leqslant n \leqslant N} A_{n}$ is transitive, and the projection $\left(a_{n}\right)_{n} \mapsto a_{N+1}$ gives an isomorphism from the stabilizer of any $x \in S_{N}$ onto $A_{N+1}$. Let $x, y \in$ $\prod_{n \in \mathbb{N}} A_{n}$, and let

$$
P_{N}=\left\{g \in G_{N+1} \mid g x^{N}=y^{N}\right\},
$$

\footnotetext{
${ }^{2}$ We usually omit the subscript on transition maps.
} 
where $x^{N}$ and $y^{N}$ are the images of $x$ and $y$ in $S_{N}$. We have to show that $\lim _{P_{N}}$ is nonempty. The observations in the first sentence show that $P_{N}$ is nonempty and that $A_{N+1}$ acts simply transitively on it. It follows that the inverse system $\left(P_{N}\right)$ satisfies (ML). Let $Q_{N}=\bigcap_{i} \operatorname{Im}\left(P_{N+i} \rightarrow P_{N}\right)$. Then each $Q_{N}$ is nonempty, and $\left(Q_{N}\right)_{N \in \mathbb{N}}$ is an inverse system with surjective transition maps. Hence, $\lim _{\longleftarrow} Q_{N}$ is (obviously) nonempty, and any element of it is an element of $\lim _{\longleftarrow} P_{N}$.

b) If $\left(A_{n}\right)$ fails (ML), then there exists an $m$ such that infinitely many of the groups

$$
B_{i} \stackrel{\text { df }}{=} \operatorname{Im}\left(A_{m+i} \rightarrow A_{m}\right)
$$

are distinct. As

$$
\lim _{i}^{1} A_{m+i} \rightarrow \underset{i}{\lim _{i}} B_{i}
$$

is surjective (see (2)), it suffices to show that $\lim ^{1} B_{i}$ is uncountable. This is accomplished by the next lemma (applied with $A=A_{m}$ ).

Lemma 1.2. Let $\cdots \supset A_{n} \supset A_{n+1} \supset \cdots$ be a sequence of subgroups of a countable group $A$. If infinitely many of the $A_{n}$ are distinct, then $\lim ^{1} A_{n}$ is uncountable.

Proof. From (1) applied to the inverse system $\left(A_{n} \hookrightarrow A\right)_{n \in \mathbb{N}}$, we obtain a bijection

$$
A \backslash\left(\lim _{\longleftarrow} A / A_{n}\right) \rightarrow \lim ^{1} A_{n} .
$$

As a map of sets, $A / A_{n+1} \rightarrow A / A_{n}$ is isomorphic to the projection map

$$
A / A_{n} \times A_{n} / A_{n+1} \rightarrow A / A_{n},
$$

and so $\lim _{\longleftarrow} A / A_{n} \approx \prod A_{n} / A_{n+1}$ (as sets), which is uncountable.

Remark 1.3. The above statements apply to inverse systems indexed by any directed set $I$ containing an infinite countable cofinal set, because such an $I$ will also contain a cofinal set isomorphic to $(\mathbb{N}, \leqslant)$.

Notes. The definition of $\lim ^{1}$ for nonabelian groups and the sequence (2) can be found in [BK72, IX, $\S 2$ ]. In the commutative case, statement a of Proposition 1.1 is proved in [Ati61] and statement b in [Gra66].

\section{Torsors}

Let $\mathrm{E}$ be a category with finite fibred products (in particular, a final object $S$ ) endowed with a topology in the sense of Grothendieck (see [BD68, ch. 2]). Thus, E is a site. By 'torsor' we mean 'right torsor'.

1.4 For a sheaf of groups $A$ on E, a right $A$-sheaf $X$, and a left $A$-sheaf $Y, X \wedge^{A} Y$ denotes the contracted product of $X$ and $Y$, i.e. the quotient sheaf of $X \times Y$ by the diagonal action of $A$, $(x, y) a=\left(x a, a^{-1} y\right)$. When $A \rightarrow B$ is a homomorphism of sheaves of groups, $X \wedge^{A} B$ is the $B$-sheaf obtained from $X$ by extension of the structure group. In this last case, if $X$ is an $A$-torsor, then $X \wedge \wedge^{A} B$ is a $B$-torsor.

1.5 For an $A$-torsor $P$ and a left $A$-sheaf $X$, define

$$
{ }^{P} X=P \wedge^{A} X \text {. }
$$

When $X$ is a sheaf of groups and $A$ acts by group homomorphisms, ${ }^{P} X$ is a sheaf of groups. For example, when we let $A$ act on itself by inner automorphisms, ${ }^{P} A$ is the inner form of $A$ defined by $P$. There is a natural left action of ${ }^{P} A$ on $P$, which makes $P$ into a left ${ }^{P} A$-torsor and induces an isomorphism

$$
{ }^{P} A \rightarrow \mathcal{A} u t_{A}(P)
$$




\section{PeRiods of ABELIAN VARIETIES}

Let $\mathrm{P}(A)$ denote the category of $A$-torsors and $H^{1}(S, A)$ the set of isomorphism classes of objects in $\mathrm{P}(A)$ (pointed by the class of the trivial torsor $A_{A}$ ).

1.6 Let $v: B \rightarrow C$ be a homomorphism of sheaves of groups on $\mathrm{E}$, and let $Q$ be a $C$-torsor. Define $\mathrm{P}(B \rightarrow C ; Q)$ to be the category whose objects are the $v$-morphisms of torsors $P \rightarrow Q$ and whose morphisms $\operatorname{Hom}\left(P \rightarrow Q, P^{\prime} \rightarrow Q\right)$ are the $B$-morphisms $P \rightarrow P^{\prime}$ giving a commutative triangle. Let $H^{1}(S, B \rightarrow C ; Q)$ denote the set of isomorphism classes in $\mathrm{P}(B \rightarrow C ; Q)$. When $Q=C_{C}$, we drop it from the notation; then $H^{1}(S, B \rightarrow C)$ is pointed by the class of $B_{B} \rightarrow C_{C}$. The category $\mathrm{P}(B \rightarrow 0)$ is canonically equivalent with the category of $B$-torsors, and so

$$
H^{1}(S, B \rightarrow 0) \cong H^{1}(S, B) .
$$

Let $A=\operatorname{Ker}(B \rightarrow C)$. Then $A$ is stable under the action of $B$ on itself by inner automorphisms, and for any object $P \rightarrow Q$ of $\mathrm{P}(B \rightarrow C ; Q)$,

$$
\mathcal{A} u t(P \rightarrow Q) \cong P \wedge^{B} A
$$

1.7 Let $v: B \rightarrow C$ be a surjective homomorphism with kernel $A$. To give a $B$-torsor $P$ with $v P$ trivialized by $e \in(v P)(S)$ amounts to giving the $A$-torsor $f^{-1}(e)$ where $f$ is the map $P \rightarrow v P$ : the natural functor

$$
\mathrm{P}(A \rightarrow 0) \rightarrow \mathrm{P}(B \rightarrow C)
$$

is an equivalence.

1.8 Let $v: B \rightarrow C$ be a homomorphism of sheaves of groups on E. A $B$-torsor $P$ allows us to twist $v$ :

$$
P_{v:}{ }^{P} B \rightarrow{ }^{P} C, \quad{ }^{P} C \stackrel{\mathrm{df}}{=} P \wedge \wedge^{B} C .
$$

Here a local section $b$ of $B$ acts on $C$ by $c \mapsto(v b) c(v b)^{-1}$. Let $Q=v P$. Then ${ }^{P} C \cong{ }^{Q} C$.

1.9 Let $v: B \rightarrow C$ be a homomorphism of sheaves of groups on $\mathrm{E}$. A $B$-torsor $P$ can be regarded as a $\left({ }^{P} B, B\right)$-bitorsor $($ see $(3))$. There is a functor

$$
\mathrm{P}\left({ }^{P} B \rightarrow{ }^{Q} C\right) \rightarrow \mathrm{P}(B \rightarrow C ; Q)
$$

sending $P^{\prime} \rightarrow Q^{\prime}$ to $P^{\prime} \wedge{ }^{P} B P \rightarrow Q^{\prime} \wedge{ }^{Q} B$. In particular, the neutral object of $\mathrm{P}\left({ }^{P} B \rightarrow{ }^{Q} C\right)$ is sent to the object $P \rightarrow Q$ of $\mathrm{P}(B \rightarrow C ; Q)$. Let $P^{\text {opp }}$ denote the $\left(B,{ }^{P} B\right)$-bitorsor with the same underlying sheaf as $P$ but with local sections $b$ and $b^{\prime}$ of $B$ and ${ }^{P} B$ acting as $\left(b, b^{\prime}\right) \cdot p=b^{-1} \cdot p \cdot b^{-1}$. The functor

$$
\mathrm{P}(B \rightarrow C ; Q) \rightarrow \mathrm{P}\left({ }^{P} B \rightarrow{ }^{Q} C\right)
$$

sending $P^{\prime} \rightarrow Q$ to $\left(P^{\prime} \rightarrow Q\right) \wedge^{B} P^{\text {opp }}$ is a quasi-inverse to the functor in (4). Therefore, both functors are equivalences of categories.

Proposition 1.10. Let

$$
1 \rightarrow A \rightarrow B \stackrel{v}{\rightarrow} C \rightarrow 0
$$

be an exact sequence of sheaves of groups on $\mathrm{E}$, and let $P \rightarrow Q$ be a $v$-morphism of torsors. There is a natural bijection

$$
H^{1}\left(S,{ }^{P} A\right) \rightarrow H^{1}(S, B \rightarrow C ; Q)
$$

sending the neutral element of $H^{1}\left(S,{ }^{P} A\right)$ to the element $[P \rightarrow Q]$ of $H^{1}(S, B \rightarrow C ; Q)$.

Proof. We can use $P$ to twist the sequence (6):

$$
1 \rightarrow{ }^{P} A \rightarrow{ }^{P} B \rightarrow{ }^{Q} C \rightarrow 1, \quad{ }^{P} A=P \wedge^{B} A .
$$


Now combine

$$
H^{1}\left(S,{ }^{P} A\right) \stackrel{1.7}{\rightarrow} H^{1}\left(S,{ }^{P} B \rightarrow{ }^{Q} C\right) \stackrel{(4)}{\rightarrow} H^{1}(S, B \rightarrow C ; Q) .
$$

Remark 1.11. If in the proposition $A$ is commutative, then the action of $B$ on $A$ factors through an action of $C$ on $A$, and so

$$
P_{A} \stackrel{\mathrm{df}}{=} P \wedge^{B} A \cong P \wedge^{B} C \wedge^{C} A \cong Q \wedge^{C} A \stackrel{\mathrm{df}}{=} Q A .
$$

Notes. The basic definitions in $\S \S 1.4$ and 1.5 are from [Gir71]. The remaining statements can be found, or are hinted at, in [Del79a, 2.4.3-2.4.4]. See also [Bre90]. (The main ideas go back to Dedecker and Grothendieck in the 1950s.)

\section{Cohomology and inverse limits}

We now fix an affine scheme $S$ and let $\mathrm{E}$ be the category of affine schemes over $S$ endowed with the FPQC topology (that for which the covering families are the finite surjective families of flat morphisms).

Throughout this subsection, $\left(G_{n}, u_{n}\right)_{n}$ is an inverse system, indexed by $(\mathbb{N}, \leqslant)$, of flat affine group schemes of finite type over $S$ with faithfully flat transition maps, and $G=\lim _{n} G_{n}$. Thus, $G$ is a flat affine group scheme over $S$.

Proposition 1.12. The map $[P] \mapsto\left(\left[P \wedge^{G} G_{n}\right]\right)_{n \geqslant 0}$

$$
H^{1}(S, G) \rightarrow \underset{n}{\lim } H^{1}\left(S, G_{n}\right)
$$

is surjective. For a $G$-torsor $P$, the fibre of the map containing $[P]$ is $\varliminf^{1} G_{n}^{\prime}(S)$ where $G_{n}^{\prime}$ is the inner form $P \wedge^{G} G_{n}$ of $G_{n}$.

Proof. A class $c$ in $\lim H^{1}\left(S, G_{n}\right)$ is represented by an inverse system

$$
P_{0} \leftarrow P_{1} \leftarrow \cdots \leftarrow P_{n} \leftarrow \cdots
$$

with $P_{n}$ a $G_{n}$-torsor. The inverse limit of this system is a $G$-torsor mapping to $c$.

Let $P^{\prime}$ and $P$ be $G$-torsors such that $P_{n}^{\prime} \approx P_{n}$ for all $n$, and choose isomorphisms $a_{n}: P_{n}^{\prime} \rightarrow P_{n}$. Consider the following diagram.

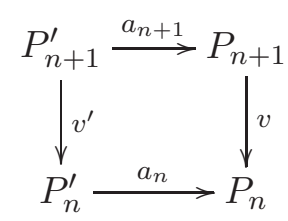

There is a unique isomorphism $b_{n}: P_{n}^{\prime} \rightarrow P_{n}$ for which the diagram commutes, i.e. such that

$$
b_{n} \circ v^{\prime}=v \circ a_{n+1} \text {. }
$$

Let $e_{n}$ be the element of $\operatorname{Aut}\left(P_{n}\right)$ such that $e_{n} \circ b_{n}=a_{n}$; then

$$
e_{n} \circ v \circ a_{n+1}=a_{n} \circ v^{\prime} \text {. }
$$

Replacing $\left(a_{n}\right)_{n \geqslant 0}$ with $\left(c_{n} \circ a_{n}\right)_{n \geqslant 0}$ replaces $\left(e_{n}\right)_{n \geqslant 0}$ with $\left(c_{n} \cdot e_{n} \cdot u^{\prime} c_{n+1}^{-1}\right)_{n \geqslant 0}$ where $u^{\prime}$ is the transition map Aut $\left(P_{n+1}\right) \rightarrow \operatorname{Aut}\left(P_{n}\right)$. Thus, the class of $\left(e_{n}\right)_{n \geqslant 0}$ in $\lim ^{1} \operatorname{Aut}\left(P_{n}\right)$ is independent of the choice of the $a_{n}$. Similarly, it depends only on the isomorphism class of $P^{\prime}$. Therefore, we have a well-defined map from the fibre containing $[P]$ to $\lim ^{1} \operatorname{Aut}\left(P_{n}\right)$, and it is straightforward to check that it is a bijection. Finally, (3) allows us to replace $\operatorname{Aut}\left(P_{n}\right)$ with $G_{n}^{\prime}(S)$.

Corollary 1.13. When the $G_{n}$ are commutative, there is an exact sequence

$$
0 \rightarrow \lim ^{1} G_{n}(S) \rightarrow H^{1}(S, G) \rightarrow \lim _{\longleftarrow} H^{1}\left(S, G_{n}\right) \rightarrow 0
$$




\section{PERIODS OF ABELIAN VARIETIES}

Proof. In this case, $G_{n}^{\prime}=G_{n}$.

Corollary 1.14. For any countable family $\left(G_{i}\right)_{i \in I}$ of flat affine group schemes,

$$
H^{1}\left(S, \prod G_{i}\right)=\prod H^{1}\left(S, G_{i}\right) .
$$

Proof. This is certainly true for finite families. Thus, we may assume that $I$ is infinite, and equals $\mathbb{N}$. Let $A_{n}=\prod_{0 \leqslant i \leqslant n} G_{i}$. For any $\prod_{i \geqslant 0} G_{i}$-torsor $P$, the projection maps ${ }^{P} A_{n}(S) \rightarrow{ }^{P} A_{n-1}(S)$ admit sections, and so are surjective. Therefore $\lim ^{1} P^{P_{n}}(S)=0$ (from statement a of Proposition 1.1), and it follows that

$$
H^{1}(S, G) \stackrel{1.12}{\cong}{\underset{\lim }{n}}_{n} H^{1}\left(S, A_{n}\right) \cong \lim _{n} \prod_{0 \leqslant i \leqslant n} H^{1}\left(S, G_{i}\right) \cong \prod_{i \geqslant 0} H^{1}\left(S, G_{i}\right) .
$$

Remark 1.15. Let $S=\operatorname{Spec}(\mathbb{Q})$. Although the maps $u_{n}: G_{n} \rightarrow G_{n-1}$ are surjective, typically the maps $G_{n}(\mathbb{Q}) \rightarrow G_{n-1}(\mathbb{Q})$ will not be. In fact, typically, the inverse system $\left(G_{n}(\mathbb{Q})\right)_{n}$ will not satisfy $(\mathrm{ML})$ and so $\lim ^{1} G_{n}(\mathbb{Q})$ will be uncountable (from statement b of Proposition 1.1). For example, consider a tower of distinct subfields of $\mathbb{Q}^{\text {al }}$,

$$
\mathbb{Q} \subset F_{1} \subset \cdots \subset F_{n-1} \subset F_{n} \subset \cdots, \quad\left[F_{n}: \mathbb{Q}\right]<\infty .
$$

There is an inverse system $\left(G_{n}, u_{n}\right)$ with surjective transition maps for which $G_{n}$ is the $\mathbb{Q}$-torus obtained from $\mathbb{G}_{m / F_{n}}$ by restriction of scalars and $u_{n}$ is the norm map. Then

$$
\left(G_{n}(\mathbb{Q}), u_{n}(\mathbb{Q})\right)_{n \in \mathbb{N}}=\left(F_{n}^{\times}, \mathrm{Nm}_{F_{n} / F_{n-1}}\right)_{n \in \mathbb{N}},
$$

which fails (ML), ${ }^{3}$ and so $\lim ^{1} G_{n}(\mathbb{Q})$ is uncountable.

\section{Comparison with Galois cohomology}

We now let $S$ be the spectrum of a field $k$, and we let $H^{1}(k,-)$ denote $H^{1}(S,-)$. Choose a separable closure $k^{\mathrm{sep}}$ of $k$, and let $\Gamma=\operatorname{Gal}\left(k^{\mathrm{sep}} / k\right)$.

Proposition 1.16. For any smooth algebraic group $N$ over $k$, there is a canonical isomorphism

$$
H^{1}(k, N) \rightarrow H^{1}\left(\Gamma, N\left(k^{\mathrm{sep}}\right)\right) .
$$

Proof. An $N$-torsor $P$ is represented by an algebraic variety over $k$, and hence acquires a point $p$ over some subfield of $k^{\mathrm{sep}}$ of finite degree over $k$. The formula

$$
\tau p=p \cdot a_{\tau}
$$

defines a continuous crossed homomorphism $a_{\tau}: \Gamma \rightarrow N\left(k^{\mathrm{sep}}\right)$ whose cohomology class is independent of the choice of $p$ and depends only on the isomorphism class of $P$. Thus, we have a well-defined map $H^{1}(k, N) \rightarrow H^{1}\left(\Gamma, N\left(k^{\mathrm{sep}}\right)\right)$, and it follows from descent theory that this is an isomorphism.

1.17 Let $N$ be a smooth algebraic group over $k$, and let $f: \Gamma \rightarrow \mathcal{A} u t(N)\left(k^{\text {sep }}\right)$ be a continuous crossed homomorphism (discrete topology on $\mathcal{A} u t(N)\left(k^{\mathrm{sep}}\right)$ ). The 'twist' of $N$ by $f$ is a smooth algebraic group ${ }_{f} N$ over $k$ such that ${ }_{f} N\left(k^{\text {sep }}\right)=N\left(k^{\text {sep }}\right)$ but with $\tau \in \Gamma$ acting according to the rule

$$
\tau * x=f(\tau) \cdot \tau x .
$$

When we let $N$ act on itself by inner automorphisms, a crossed homomorphism $f: \Gamma \rightarrow N\left(k^{\mathrm{sep}}\right)$ defines a twist ${ }_{f} N$ of $N$ with $\tau \in \Gamma$ acting on ${ }_{f} N\left(k^{\text {sep }}\right)$ by

$$
\tau * x=f(\tau) \cdot \tau x \cdot f(\tau)^{-1} .
$$

\footnotetext{
${ }^{3}$ To see this, use that, for a finite extension $E / F$ of number fields and a finite prime $v$ of $F$, ord $\left(\mathrm{Nm}^{\times}\right)$is the ideal in $\mathbb{Z}$ generated by the residue class degrees of the primes of $E$ lying over $v$.
} 


\section{J. S. MiLnE}

1.18 Let $G=\lim \left(G_{n}, u_{n}\right)$ be as in the preceding subsection but with $G_{n}$ now a smooth algebraic group over $k$, and define $H_{\mathrm{cts}}^{1}(\Gamma, G)$ be the cohomology set computed using crossed homomorphisms $\Gamma \rightarrow G\left(k^{\mathrm{sep}}\right)$ that are continuous for the profinite topology on $\Gamma$ and the inverse limit topology on $G\left(k^{\mathrm{sep}}\right)=\lim G_{n}\left(k^{\mathrm{sep}}\right)$ (discrete topology on $G_{n}\left(k^{\mathrm{sep}}\right)$ ). Thus, giving a continuous crossed homomorphism $f: \Gamma \rightarrow G\left(k^{\text {sep }}\right)$ amounts to giving a compatible family of continuous crossed homomorphisms $f_{n}: \Gamma \rightarrow G_{n}\left(k^{\mathrm{sep}}\right)$.

Proposition 1.19. The map

$$
H_{\mathrm{cts}}^{1}(\Gamma, G) \rightarrow \lim _{\longleftarrow} H^{1}\left(\Gamma, G_{n}\right)
$$

sending $[f]$ to $\left(\left[f_{n}\right]\right)_{n \geqslant 0}$ is surjective. The fibre of the map containing $[f]$ equals $\varliminf^{1} G_{n}^{\prime}(k)$ where $G_{n}^{\prime}={ }_{f} G_{n}$.

Proof. Each class $c$ in $\lim _{\longleftarrow} H^{1}\left(S, G_{n}\right)$ is represented by a family $\left(f_{n}\right)_{n \geqslant 0}$ of crossed homomorphisms, which can be chosen so that $f_{n-1}=u_{n} \circ f_{n}$. The $f_{n}$ define a continuous crossed homomorphism $f: \Gamma \rightarrow G\left(k^{\mathrm{sep}}\right)$ mapping to $c$.

Let $f^{\prime}$ and $f$ be continuous crossed homomorphisms such that $f_{n}^{\prime} \sim f_{n}$ for all $n$, and choose $a_{n} \in G_{n}\left(k^{\text {sep }}\right)$ in such a way that

$$
f_{n}^{\prime}(\tau)=a_{n}^{-1} \cdot f_{n}(\tau) \cdot \tau a_{n}
$$

Define $e_{n} \in G_{n}\left(k^{\text {sep }}\right)$ by the equation

$$
e_{n} \cdot u a_{n+1}=a_{n}
$$

On applying $u$ to Equation $(9)_{n+1}$, we obtain the equation

$$
f_{n}^{\prime}(\tau)=\left(u a_{n+1}\right)^{-1} \cdot f_{n}(\tau) \cdot \tau\left(u a_{n+1}\right)
$$

or

$$
f_{n}^{\prime}(\tau)=a_{n}^{-1} \cdot e_{n} \cdot f_{n}(\tau) \cdot \tau e_{n}^{-1} \cdot \tau a_{n}
$$

On comparing this with (9), we find that

$$
e_{n}=f_{n}(\tau) \cdot \tau e_{n} \cdot f_{n}(\tau)^{-1},
$$

i.e. that

$$
e_{n} \in\left({ }_{f} G_{n}\right)\left(k^{\mathrm{sep}}\right)^{\Gamma}=\left({ }_{f} G_{n}\right)(k) .
$$

The element $a_{n}$ can be replaced by $c_{n} \cdot a_{n}$, where $c_{n}$ is any element of $\left({ }_{f} G_{n}\right)(k)$. When this is done for each $n,\left(e_{n}\right)_{n \geqslant 0}$ is replaced by $\left(c_{n} \cdot e_{n} \cdot\left(u c_{n+1}\right)^{-1}\right)_{n \geqslant 0}$. Thus, the class of $\left(e_{n}\right)$ in $\lim ^{1}\left({ }_{f} G_{n}\right)(k)$ is independent of the choice of the $a_{n}$. Similarly, it depends only on the cohomology class of $f^{\prime}$. Therefore, we have a well-defined map from the fibre containing $[f]$ to $\lim ^{1}\left({ }_{f} G_{n}\right)(k)$, and it is straightforward to check that this is a bijection.

Proposition 1.20. There is a canonical isomorphism of pointed sets

$$
H^{1}(k, G) \rightarrow H_{\mathrm{cts}}^{1}(\Gamma, G) .
$$

Proof. Let $P$ be a $G$-torsor, and let $P_{n}=u_{n} P$. Then $P\left(k^{\text {sep }}\right)=\lim P_{n}\left(k^{\mathrm{sep}}\right)$, which, because the maps $P_{n+1}\left(k^{\mathrm{sep}}\right) \rightarrow P_{n}\left(k^{\mathrm{sep}}\right)$ are surjective, is nonempty. Choose a $\overleftarrow{p} \in P\left(k^{\mathrm{sep}}\right)$. Then the formula

$$
\tau p=p \cdot f(\tau)
$$

defines a continuous crossed homomorphism $f: \Gamma \rightarrow G\left(k^{\mathrm{sep}}\right)$ whose cohomology class is independent of the choice of $p$ and of the choice of $P$ in its isomorphism class. Therefore, we have a well-defined map $H^{1}(k, G) \rightarrow H_{\text {cts }}^{1}(\Gamma, G)$. Since this map is compatible with the maps in Propositions 1.12 and 1.19, they, together with Proposition 1.16, show that it is a bijection. 


\section{PeRiods of ABELIAN VARIETIES}

Remark 1.21. Let $S$ be the spectrum of a field. The following conditions on an affine group scheme $G$ over $S$ are equivalent:

a) the set of closed normal subgroup schemes $H \subset G$ such that $G / H$ is of finite type over $S$ is infinite and countable;

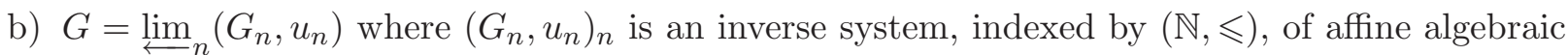
groups over $S$ with surjective transition maps.

An affine group scheme $G$ satisfying these conditions will be said to be separable. When $G$ is separable, any inverse system satisfying condition b is cofinal in the inverse system of all algebraic quotients of $G$. Therefore, the former can be replaced by the latter, which makes Proposition 1.19 more canonical.

Notes. Proposition 1.16 is a standard result. In the commutative case, Proposition 1.19 is proved in [Tat76].

\section{Application to periods}

Let $\operatorname{Mot}(\mathbb{Q})$ denote the category of motives based on all smooth projective varieties over $\mathbb{Q}$. Let $\mathrm{CM}(\mathbb{Q})$ be the Tannakian subcategory of $\operatorname{Mot}(\mathbb{Q})$ generated by the zero-dimensional varieties over $\mathbb{Q}$ and the abelian varieties of CM-type (see $\S 2)$. Let $G^{\mathrm{Mot}}=\mathcal{A} u t^{\otimes}\left(\omega_{\mathrm{B}}\right)$ and $P^{\mathrm{Mot}}=\mathcal{I}$ som ${ }^{\otimes}\left(\omega_{\mathrm{B}}, \omega_{\mathrm{dR}}\right)$, and define $G^{\mathrm{CM}}$ and $P^{\mathrm{CM}}$ similarly. From the inclusion $\mathrm{CM}(\mathbb{Q}) \subset \operatorname{Mot}(\mathbb{Q})$, we obtain a faithfully flat homomorphism $G^{\mathrm{Mot}} \rightarrow G^{\mathrm{CM}}$.

Theorem 1.22. If the kernel of $G^{\mathrm{Mot}} \rightarrow G^{\mathrm{CM}}$ is an inverse limit of simply connected semisimple groups, then the isomorphism class of $P^{\mathrm{Mot}} \rightarrow P^{\mathrm{CM}}$ in $\mathrm{P}\left(G^{\mathrm{Mot}} \rightarrow G^{\mathrm{CM}} ; P^{\mathrm{CM}}\right)$ is uniquely determined by its class over $\mathbb{R}$.

Proof. Let $G=\operatorname{Ker}\left(G^{\mathrm{Mot}} \rightarrow G^{\mathrm{CM}}\right)$. The condition on $G$ implies that it is, in fact, a product of semisimple groups each of which is simply connected. Moreover, the product is countable because $\operatorname{Mot}(\mathbb{Q})$ is generated as a Tannakian category by a countable set of varieties. According to Proposition 1.10 , the isomorphism classes in $\mathrm{P}\left(G^{\text {Mot }} \rightarrow G^{\mathrm{CM}} ; P^{\mathrm{CM}}\right)$ are classified by $H^{1}\left(\mathbb{Q}, G^{\prime}\right)$ where $G^{\prime}=P^{\text {Mot }} \wedge^{G^{\text {Mot }}} G$. As $G^{\prime}$ is a form of $G$, it also is a countable product of simply connected semisimple groups, and so the proposition follows from Corollary 1.14 and the theorem of Kneser, Harder, and Chernousov (see Lemma 3.6 below).

Remark 1.23. a) The group $G^{\text {Mot }}$ is proreductive because $\operatorname{Mot}(\mathbb{Q})$ is semisimple. If Deligne's hope [Del79b] that every Hodge class is an absolute Hodge class is true, then $\left(G^{\text {Mot }}\right)^{\circ}$ is the group attached to the category of motives over $\mathbb{Q}^{\text {al }}$ (see $[\mathrm{DM} 82,6.22,6.23]$, where the hypothesis was inadvertently omitted); moreover, the group $G$ in the above proof is the kernel of the canonical homomorphism from $\left(G^{\text {Mot }}\right)^{\circ}$ to the Serre group [DM82, p. 220] and it is the derived group of $\left(G^{\text {Mot }}\right)^{\circ}$; it is therefore an inverse limit of semisimple groups.

b) It is generally hoped that the derived group of $\left(G^{\mathrm{Mot}}\right)^{\circ}$ is simply connected; see the question in $[$ Ser94, 8.1].

c) Blasius and Borovoi [BB99, 1.6] assert that the analogue of their Theorem 1.5 holds for all motives if the derived group of $\left(G^{\mathrm{Mot}}\right)^{\circ}$ is simply connected. Theorem 1.22 replaces this assertion, which is unproven (and seems unlikely to be true).

Remark 1.24. Let $G=\operatorname{Ker}\left(G^{\mathrm{Mot}} \rightarrow G^{\mathrm{CM}}\right)$, and let $H \subset G$ be the intersection of the kernels of the homomorphisms from $G$ onto simply connected semisimple algebraic groups over $\mathbb{Q}$. Let $\operatorname{Mot}^{H}(\mathbb{Q})$ be the subcategory of $\operatorname{Mot}(\mathbb{Q})$ of objects on which $H$ acts trivially. Then, with the obvious notations, the isomorphism class of $P^{\mathrm{Mot}^{H}} \rightarrow P^{\mathrm{CM}}$ in $\mathrm{P}\left(G^{\mathrm{Mot}^{H}} \rightarrow G^{\mathrm{CM}} ; P^{\mathrm{CM}}\right)$ is uniquely determined by its class over $\mathbb{R}$. Remark 1.23 indicates that it is reasonable to hope that $H=0$. 


\section{J. S. Milne}

\section{Periods of abelian varieties with complex multiplication}

\section{Periods of zero-dimensional varieties}

The category of motives based on the zero-dimensional varieties over $\mathbb{Q}$ is denoted $\operatorname{Art}(\mathbb{Q})$ and is called the category of Artin motives over $\mathbb{Q}$. The Betti fibre functor $\omega_{\mathrm{B}}$ defines an equivalence of $\operatorname{Art}(\mathbb{Q})$ with the category of continuous representations of $\Gamma$ on finite-dimensional $\mathbb{Q}$-vector spaces $[\mathrm{DM} 82,6.17]$ from which it follows that $\mathcal{A} u t^{\otimes}\left(\omega_{B}\right)$ is the constant profinite group scheme $\Gamma$ with $\Gamma(\mathbb{Q})=\Gamma\left(\mathbb{Q}^{\text {al }}\right)=\Gamma$. The de Rham fibre functor is $h X \mapsto \Gamma\left(X, \mathcal{O}_{X}\right)$, from which the next statement follows easily.

Theorem 2.1. Let $P^{\text {Art }}$ be the period torsor for $\operatorname{Art}(\mathbb{Q})$. Then $P^{\text {Art }} \cong \operatorname{Spec} \mathbb{Q}^{\text {al }}$ with its natural action of $\Gamma$, and $p^{\text {Art }}$ is the obvious $\mathbb{Q}^{\text {al }}$-point of Spec $\mathbb{Q}^{\text {al }}$. Thus, the period point $p^{\text {Art }}$ has coordinates in $\mathbb{Q}^{\text {al }}$, and the cocycle corresponding to the pair ( $\left.P^{\text {Art }}, p^{\text {Art }}\right)$ is the crossed homomorphism $\Gamma \rightarrow \Gamma\left(\mathbb{Q}^{\mathrm{al}}\right)$, $\tau \mapsto \tau$.

Note that the theorem determines the pair $\left(P^{\text {Art }}, p^{\text {Art }}\right)$ uniquely up to a unique isomorphism.

Throughout this section, we use $f$ to denote the (crossed) homomorphism in the theorem.

\section{Notations for tori}

For a finite étale $\mathbb{Q}$-algebra $A$, let

$$
\begin{aligned}
\Sigma_{A / \mathbb{Q}} & =\operatorname{Hom}_{\mathbb{Q} \text {-alg }}\left(A, \mathbb{Q}^{\text {al }}\right), \\
\Gamma_{A / \mathbb{Q}} & =\operatorname{Aut}_{\mathbb{Q} \text {-alg }}(A),
\end{aligned}
$$

and let $\left(\mathbb{G}_{m}\right)_{A / \mathbb{Q}}$ be the torus over $\mathbb{Q}$ obtained from $\mathbb{G}_{m / A}$ by restriction of scalars. Thus

$$
\left(\mathbb{G}_{m}\right)_{A / \mathbb{Q}}(R)=(A \otimes R)^{\times}
$$

for all $\mathbb{Q}$-algebras $R$. For an infinite field extension $K / k$,

$$
\left(\mathbb{G}_{m}\right)_{K / k}=\lim _{\longleftarrow}\left(\mathbb{G}_{m}\right)_{K^{\prime} / \mathbb{Q}}, \quad K^{\prime} \subset K, \quad\left[K^{\prime}: \mathbb{Q}\right]<\infty .
$$

2.2 There is an equivalence $T \mapsto X^{*}(T)=_{\mathrm{df}} \operatorname{Hom}\left(T_{/ \mathbb{Q}^{a l}}, \mathbb{G}_{m}\right)$ from the category of tori over $\mathbb{Q}$ to the category of finitely generated free $\mathbb{Z}$-modules endowed with a continuous left action of $\Gamma$.

2.3 There is an equivalence $A \mapsto \Sigma_{A / \mathbb{Q}}$ from the category of finite étale $\mathbb{Q}$-algebras to the category of finite sets endowed with a continuous left action of $\Gamma$. A quasi-inverse is provided by

$$
\Sigma \mapsto A(\Sigma) \stackrel{\text { df }}{=} \operatorname{Hom}_{\Gamma}\left(\Sigma, \mathbb{Q}^{\text {al }}\right) .
$$

If $\Sigma \leftrightarrow A$, then the decomposition $\Sigma=\amalg \Sigma_{i}$ of $\Sigma$ into orbits corresponds to the decomposition $A=\prod A\left(\Sigma_{i}\right)$ of $A$ into a product of fields. If $\Gamma$ acts transitively on $\Sigma$, then the choice of an $e \in \Sigma$ determines isomorphisms $\Gamma / \Gamma_{e} \rightarrow \Sigma$ and $A(\Sigma) \rightarrow\left(\mathbb{Q}^{\text {al }}\right)^{\Gamma_{e}}$. Here $\Gamma_{e}=\{\tau \in \Gamma \mid \tau e=e\}$.

2.4 On combining these equivalences, we see that there is a fully faithful functor $\Sigma \mapsto T^{\Sigma}$ from the category of finite sets endowed with a continuous left $\Gamma$-action to the category of tori, for which $T^{\Sigma}=\left(\mathbb{G}_{m}\right)_{A(\Sigma) / \mathbb{Q}}, X^{*}\left(T^{\Sigma}\right)=\mathbb{Z}[\Sigma]$ (free $\mathbb{Z}$-module on $\Sigma$ with the natural left action of $\Gamma$ ), and $T^{\Sigma_{1} \sqcup \Sigma_{2}}=T^{\Sigma_{1}} \times T^{\Sigma_{2}}$.

\section{Abelian varieties with complex multiplication}

For an abelian variety $A$ defined over a subfield $k$ of $\mathbb{C}$, the Mumford-Tate group of $A$ is

$$
\begin{gathered}
\operatorname{MT}(A)=\mathcal{A} u t^{\otimes}\left(\omega_{\mathrm{B}} \mid\left\langle A_{/ \mathbb{C}}\right\rangle^{\otimes}\right), \\
1158
\end{gathered}
$$




\section{PERIODS OF ABELIAN VARIETIES}

where $\left\langle A_{/ \mathbb{C}}\right\rangle^{\otimes}$ is the category of motives based on $A_{/ \mathbb{C}}$ and the projective spaces. There is a canonical cocharacter $\mu^{A}$ of $\operatorname{MT}(A)$ that splits the Hodge filtration on $H_{\mathrm{B}}^{1}(A)$. When $\operatorname{MT}(A)$ is commutative (hence a torus), we say that $A$ is of $C M$-type, ${ }^{4}$ and we define the reflex field of $A$ to be the field of definition of $\mu^{A}$. It is a CM-subfield of $\mathbb{C}$.

For subfields $k, K$ of $\mathbb{C}$ with $K$ a CM-field, we define $\mathrm{CM}^{K}(k)$ to be the category of motives based on:

- the abelian varieties of CM-type over $k$ with reflex field contained in $K$;

- the projective spaces; and

- the zero-dimensional varieties.

When $K=\mathbb{Q}^{\mathrm{cm}}$, the composite of all CM-subfields of $\mathbb{Q}^{\text {al }}$, we omit it from the notation.

\section{The category $\mathrm{CM}(\mathbb{C})$}

2.5 Let $K$ be a CM-subfield of $\mathbb{C}$. With the notation of $\oint 2.4,\left(\mathbb{G}_{m}\right)_{K / \mathbb{Q}}=T^{\Sigma_{K / \mathbb{Q}}}$ and $X^{*}\left(\left(\mathbb{G}_{m}\right)_{K / \mathbb{Q}}\right)=\mathbb{Z}\left[\Sigma_{K / \mathbb{Q}}\right]$. The group $S^{K}=\mathcal{A} u t^{\otimes}\left(\omega_{B} \mid \mathrm{CM}^{K}(\mathbb{C})\right)$ is called the Serre group for $K$. When $K$ has finite degree over $\mathbb{Q}, S^{K}$ is the quotient of $\left(\mathbb{G}_{m}\right)_{K / \mathbb{Q}}$ such that

$$
X^{*}\left(S^{K}\right)=\left\{n \in \mathbb{Z}\left[\Sigma_{K / \mathbb{Q}}\right] \mid n+\iota n=\text { constant }\right\} .
$$

Thus, there is an exact sequence

$$
0 \rightarrow\left(\mathbb{G}_{m}\right)_{F / \mathbb{Q}} \rightarrow\left(\mathbb{G}_{m}\right)_{K / \mathbb{Q}} \times \mathbb{G}_{m} \rightarrow S^{K} \rightarrow 0
$$

where $F$ is the largest totally real subfield of $K$. We have

$$
S=\lim _{\overleftarrow{K \in \mathcal{K}}} S^{K}
$$

where $\mathcal{K}$ is the set of all CM-subfields of $\mathbb{C}$ finite and Galois over $\mathbb{Q}$.

2.6 For an abelian variety $A$ over $\mathbb{C}$, there is a canonical surjection $S \rightarrow \operatorname{MT}(A)$, which factors through $S^{K}$ if and only if $A$ has reflex field contained in $K$.

Notes. For more on Mumford-Tate groups, see [Del82a, § 3].

\section{The category $\mathrm{CM}\left(\mathbb{Q}^{\mathrm{al}}\right)$}

2.7 The functor from the category of abelian varieties of CM-type up to isogeny over $\mathbb{Q}^{\text {al }}$ to the similar category over $\mathbb{C}$ is an equivalence. For any CM-subfield $K$ of $\mathbb{C}$, the functor 'extension of scalars' $\mathrm{CM}^{K}\left(\mathbb{Q}^{\mathrm{al}}\right) \rightarrow \mathrm{CM}^{K}(\mathbb{C})$ is an equivalence of tensor categories.

\section{The period torsor}

2.8 Let $K$ be a CM-subfield of $\mathbb{C}$, Galois over $\mathbb{Q}$. Then $\Gamma$ acts on the terms of the sequence (10) in such a way that the action on the sequence of $\mathbb{Q}$-points

$$
1 \rightarrow F^{\times} \rightarrow K^{\times} \times \mathbb{Q}^{\times} \rightarrow S^{K}(\mathbb{Q}) \rightarrow 1
$$

is the obvious one. Let

The tensor functors

$$
G_{\mathrm{B}}^{\mathrm{CM}, K}=\mathcal{A} u t^{\otimes}\left(\omega_{\mathrm{B}} \mid \mathrm{CM}^{K}(\mathbb{Q})\right) .
$$

$$
\mathrm{CM}^{K}\left(\mathbb{Q}^{\mathrm{al}}\right) \leftarrow \mathrm{CM}^{K}(\mathbb{Q}) \leftarrow \operatorname{Art}(\mathbb{Q})
$$

\footnotetext{
${ }^{4}$ Other authors, and this author at other times, say that $A$ is potentially of CM-type.
} 


\section{J. S. MiLnE}

define homomorphisms

$$
1 \rightarrow S^{K} \rightarrow G_{\mathrm{B}}^{\mathrm{CM}, K} \stackrel{v}{\rightarrow} \Gamma \rightarrow 1
$$

This sequence is exact and the action of $\Gamma$ on $S^{K}$ it defines is that described above [Del82b, Lemmas 1 and 2].

2.9 Let $P^{\mathrm{CM}, K}$ be the period torsor for $\mathrm{CM}^{K}(\mathbb{Q})$. The second functor in $(11)$ defines a $v$-morphism $P^{\mathrm{CM}, K} \rightarrow P^{\text {Art }}$, and since $P^{\text {Art }}$ is known (Theorem 2.1), in order to determine $P^{\mathrm{CM}, K}$ it suffices to determine $P^{\mathrm{CM}, K} \rightarrow P^{\text {Art }}$ as an object of $\mathrm{P}\left(G_{\mathrm{B}}^{\mathrm{CM}, K} \rightarrow \Gamma ; P^{\text {Art }}\right)$. The first step is to correctly identify the cohomology group classifying the isomorphism classes of objects in this category.

Proposition 2.10. Let $f: \Gamma \rightarrow \Gamma(\mathbb{Q})$ be as in Theorem 2.1, and let ${ }_{f} S^{K}$ be the twist of $S^{K}$ by $f$. There is a natural one-to-one correspondence between the set of isomorphism classes in $\mathrm{P}\left(G_{\mathrm{B}}^{\mathrm{CM}, K} \rightarrow \Gamma ; P^{\text {Art }}\right)$ and $H^{1}\left(\mathbb{Q}, f_{f} S^{K}\right)($ FPQC cohomology $)$.

Proof. Apply Proposition 1.10, Remark 1.11, and Theorem 2.1.

In particular, when $K=\mathbb{Q}^{\mathrm{cm}}$, the isomorphism classes of objects $P \rightarrow P^{\text {Art }}$ are classified by the group $H^{1}\left(\mathbb{Q},{ }_{f} S\right)$. According to Corollary 1.13 , there is an exact sequence

$$
0 \rightarrow \lim _{K \in \mathcal{K}}{ }_{f} S^{K}(\mathbb{Q}) \rightarrow H^{1}\left(\mathbb{Q}, f_{f} S\right) \rightarrow \lim _{K \in \mathcal{K}} H^{1}\left(\mathbb{Q},{ }_{f} S^{K}\right) \rightarrow 0
$$

We shall show the following proposition.

Proposition 2.11. The group $\varliminf_{K \in \mathcal{K}} H^{1}\left(\mathbb{Q},{ }_{f} S^{K}\right)=0$, but $\varliminf_{K \in \mathcal{K}}^{1} S^{K}(\mathbb{Q})$ is uncountable. Therefore,

$$
H^{1}\left(\mathbb{Q},{ }_{f} S\right) \cong \lim _{K \in \mathcal{K}}{ }_{f} S^{K}(\mathbb{Q})
$$

and is uncountable.

Twisting $\left(\mathbb{G}_{m}\right)_{L / \mathbb{Q}}$

2.12 Let $L$ be a finite extension of $\mathbb{Q}$, and let $T=\left(\mathbb{G}_{m}\right)_{L / \mathbb{Q}}$.

a) The (left) action of $\tau \in \Gamma$ on $\Sigma_{L / \mathbb{Q}}, \tau \sigma=\tau \circ \sigma$, corresponds to the natural (left) action of $\Gamma$ on $X^{*}(T)=\mathbb{Z}\left[\Sigma_{L / \mathbb{Q}}\right]($ see $\S 2.4)$.

b) Assume $L \subset \mathbb{Q}^{\text {al }}$ and is Galois over $\mathbb{Q}$. Identify $\Sigma_{L / \mathbb{Q}}$ with $\Gamma_{L / \mathbb{Q}}$. There is then a natural (left) action of $\Gamma$ on $T$ (as a torus over $\mathbb{Q}$ ), which defines a (left) action of $\Gamma$ on $X^{*}(T)=\mathbb{Z}\left[\Gamma_{L / \mathbb{Q}}\right]$. The former gives the obvious action of $\Gamma$ on $T(\mathbb{Q})=L^{\times}$, and the latter corresponds to the (left) action of $\tau \in \Gamma$ on $\Gamma_{L / \mathbb{Q}}, \tau \sigma=\sigma \circ \tau^{-1}$.

Lemma 2.13. For a subfield $L$ of $\mathbb{Q}^{\text {al }}$, finite and Galois over $\mathbb{Q}$, let ${ }_{f} \Gamma_{L / \mathbb{Q}}$ denote the twist of $\Gamma_{L / \mathbb{Q}}$ by $f$ (so that $\tau \in \Gamma$ acts by $\tau \sigma=\tau \circ \sigma \circ \tau^{-1}$ [Ser64, 5.3]), and let

$$
B(L)=A\left({ }_{f} \Gamma_{L / \mathbb{Q}}\right) .
$$

Then

$$
f\left(\mathbb{G}_{m}\right)_{L / \mathbb{Q}} \cong\left(\mathbb{G}_{m}\right)_{B(L) / \mathbb{Q}}
$$

Proof. Clearly, $f\left(\mathbb{G}_{m}\right)_{L / \mathbb{Q}} \cong T^{f} \Gamma_{L / \mathbb{Q}}$, which equals $\left(\mathbb{G}_{m}\right)_{A\left({ }_{f} \Gamma_{L / \mathbb{Q}}\right) / \mathbb{Q}}$.

Note that the orbits of $\Gamma$ acting on ${ }_{f} \Gamma_{L / \mathbb{Q}}$ are the conjugacy classes $C$ in $\Gamma_{L / \mathbb{Q}}$, and so (from $\S \S 2.3$ and 2.4)

$$
B(L)=\prod_{C} A(C), \quad\left(\mathbb{G}_{m}\right)_{B(L) / \mathbb{Q}}=\prod_{C} T^{C}
$$




\section{PERIODS OF ABELIAN VARIETIES}

For any $\sigma \in C, A(C) \cong L^{Z(\sigma)}$ where $Z(\sigma)$ is the centralizer of $\sigma$ in $\Gamma_{L / \mathbb{Q}}$.

The tori ${ }_{f} S^{K}$ and ${ }_{f} \bar{S}^{K}$

Let $K \in \mathcal{K}$. Let $w: \mathbb{G}_{m} \rightarrow S^{K}$ be the weight homomorphism, and let $\bar{S}^{K}=S^{K} / w\left(\mathbb{G}_{m}\right)$. Then

$$
X^{*}\left(\bar{S}^{K}\right)=\left\{n \in \mathbb{Z}\left[\Sigma_{K}\right] \mid n+\iota n=1\right\},
$$

and there is an exact sequence

$$
1 \rightarrow\left(\mathbb{G}_{m}\right)_{F / \mathbb{Q}} \rightarrow\left(\mathbb{G}_{m}\right)_{K / \mathbb{Q}} \rightarrow \bar{S}^{K} \rightarrow 1
$$

The group $\Gamma$ acts on the exact sequence (13), and so we can twist the sequence by $f$ to obtain an exact sequence

$$
1 \rightarrow{ }_{f}\left(\mathbb{G}_{m}\right)_{F / \mathbb{Q}} \rightarrow{ }_{f}\left(\mathbb{G}_{m}\right)_{K / \mathbb{Q}} \rightarrow{ }_{f} \bar{S}^{K} \rightarrow 1 .
$$

Lemma 2.14. If $K$ contains a quadratic imaginary field $k$, then

$$
{ }_{f} \bar{S}^{K} \approx\left(\mathbb{G}_{m}\right)_{B(F) / \mathbb{Q}}
$$

where $F$ is the largest totally real subfield of $K$.

Proof. As

$$
\Gamma_{K / \mathbb{Q}}=\Gamma_{F / \mathbb{Q}} \times \Gamma_{k / \mathbb{Q}}, \quad \Gamma_{k / \mathbb{Q}}=\{1, \iota\},
$$

for each conjugacy class $C$ in $\Gamma_{F / \mathbb{Q}}$, there are exactly two conjugacy classes in $\Gamma_{K / \mathbb{Q}}$ mapping to it, namely,

$$
C_{1}=\{(\tau, 1) \mid \tau \in C\}, \quad C_{\iota}=\{(\tau, \iota) \mid \tau \in C\} .
$$

Therefore, (14) can be written

$$
1 \rightarrow \prod_{C} T^{C} \rightarrow \prod_{C}\left(T^{C_{1}} \times T^{C_{\iota}}\right) \rightarrow{ }_{f} \bar{S}^{K} \rightarrow 1
$$

(product over the conjugacy classes in $\Gamma_{F / \mathbb{Q}}$ ). Since

$$
C \cong C_{1} \cong C_{\iota} \quad(\text { as } \Gamma \text {-sets })
$$

each of the maps

is an isomorphism.

$$
\prod_{C} T^{C} \rightarrow \prod_{C} T^{C_{1}}, \quad \prod_{C} T^{C_{1}} \rightarrow{ }_{f} \bar{S}^{K}
$$

\section{The group $\varliminf_{K \in \mathcal{K}} H^{1}\left(\mathbb{Q},{ }_{f} S^{K}\right)$}

Proposition 2.15 [Win90, 1.3]. If $K$ contains a quadratic imaginary number field, then

$$
H^{1}\left(\mathbb{Q}, S^{K}\right)=0
$$

Proof. From the exact sequence

$$
1 \rightarrow \mathbb{G}_{m} \rightarrow{ }_{f} S^{K} \rightarrow{ }_{f} \bar{S}^{K} \rightarrow 1
$$

we see that it suffices to show that $H^{1}\left(\mathbb{Q},{ }_{f} \bar{S}^{K}\right)=0$, but this follows from Lemma 2.14 and Hilbert's Theorem 90 .

Corollary 2.16. The group $\varliminf_{K \in \mathcal{K}} H^{1}\left(\mathbb{Q},{ }_{f} \bar{S}^{K}\right)=0$.

Proof. The CM-fields satisfying the hypothesis of the proposition are cofinal in $\mathcal{K}$.

Remark 2.17. In [Win90, p. 3] an example where the proposition is false without the hypothesis on $K$ is shown. 


\section{J. S. MiLne}

The group $\lim _{K \in \mathcal{K}}^{1}{ }^{1} S^{K}(\mathbb{Q})$

Proposition 2.18. The group $\lim _{K \in \mathcal{K}}^{1} S^{K}(\mathbb{Q})$ is uncountable.

From (2) applied to the cohomology sequence of (15), we see that

$$
{\underset{K}{K \in \mathcal{K}}}_{f} S^{K}(\mathbb{Q}) \stackrel{\cong}{\rightarrow}{\underset{K}{K \in \mathcal{K}}}_{f}^{1} \bar{S}^{K}(\mathbb{Q})
$$

and so we compute the second group.

Lemma 2.19. Let $\mathcal{F}$ be the set of totally real subfields of $\mathbb{C}$ that are finite and Galois over $\mathbb{Q}$. Then

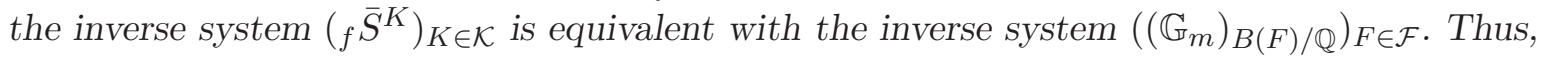

$$
\lim _{K \in \mathcal{K}}{ }_{f} \bar{S}^{K}(\mathbb{Q}) \approx \lim _{F \in \mathcal{F}}^{1} B(F)^{\times}
$$

(on the right, the transition maps are the norm maps).

Proof. Fix a quadratic imaginary field $k$. The fields $K \in \mathcal{K}$ containing $k$ form a cofinal set. Once $k$ has been fixed, the isomorphism in Lemma 2.14 becomes canonical. In particular, it is natural for the norm maps.

Note that the isomorphism in the lemma depends only on the choice of $k$.

Example 2.20. If $\Gamma_{F / \mathbb{Q}}=\operatorname{Gal}(F / \mathbb{Q})$ is commutative, $B(F)$ is a product of copies of $\mathbb{Q}$ indexed by the elements of $\Gamma_{F / \mathbb{Q}}$. Thus, $\left(\mathbb{G}_{m}\right)_{B(F) / \mathbb{Q}} \approx \mathbb{G}_{m}^{[F: \mathbb{Q}]}$.

Example 2.21. Let $F=F_{1} \cdot F_{2}$ with $F_{1}, F_{2} \in \mathcal{F}$ and $F_{1} \cap F_{2}=\mathbb{Q}$. Then

$$
\operatorname{Gal}(F / \mathbb{Q}) \cong \operatorname{Gal}\left(F_{1} / \mathbb{Q}\right) \times \operatorname{Gal}\left(F_{2} / \mathbb{Q}\right)
$$

and a conjugacy class $C$ in $\operatorname{Gal}\left(F_{1} / \mathbb{Q}\right)$ is the image of the conjugacy class $C \times\{1\}$ in $\operatorname{Gal}(F / \mathbb{Q})$. Therefore, $B\left(F_{1}\right)$ is a direct factor of $B(F)$, and so the norm map

$$
B(F)^{\times} \rightarrow B\left(F_{1}\right)^{\times}
$$

is surjective.

Example 2.22. Fix an odd prime $l$, and write $C_{l}$ for any cyclic group of order $l$. Define $G$ to be the semidirect product $N \rtimes_{\theta} Q$ of $N=C_{l} \times C_{l}$ (generators $a, b$ ) with $Q=C_{l}$ (generator $c$ ) relative to the homomorphism $\theta: C_{l} \rightarrow \operatorname{Aut}\left(C_{l} \times C_{l}\right)$ for which

$$
\theta\left(c^{i}\right)=\left(\begin{array}{ll}
1 & 0 \\
i & 1
\end{array}\right), \quad \text { i.e. } \theta\left(c^{i}\right)(a)=a b^{i}, \quad \theta\left(c^{i}\right)(b)=b .
$$

Then $G$ has generators $a, b, c$, and relations

$$
a^{l}=b^{l}=c^{l}=1, \quad a b=c a c^{-1}, \quad[b, a]=1=[b, c] .
$$

All elements $\neq 1$ in $G$ have order $l$, and the centre of $G$ is $\langle b\rangle$.

The inverse image under $G \rightarrow Q$ of the conjugacy class $\{c\}$ breaks up into $l$ conjugacy classes, namely,

$$
\left\{a^{j} c, b a^{j} c, \ldots, b^{l-1} a^{j} c\right\}, \quad 0 \leqslant j \leqslant l-1
$$

(because $a^{-1} c a=b c$ ). The centralizer of $a^{j} c$ in $G$ is $\left\langle b, a^{j} c\right\rangle$, which has order $l^{2}$.

Let $E$ be an extension of $\mathbb{Q}$ with Galois group $G$, and let $F_{0}=E^{N}$. Then from Example 2.20

$$
B\left(F_{0}\right) \cong F_{0}^{\{1\}} \times F_{0}^{\{c\}} \times \cdots \times F_{0}^{\left\{c^{l-1}\right\}}, \quad F_{0}^{\left\{c^{i}\right\}}=\mathbb{Q},
$$




\section{PeRiods of ABELIAN VARIETIES}

and the inverse image of $F_{0}^{\{c\}}$ in $B(E)$ under the norm map is

$$
E^{\langle b, c\rangle} \times E^{\langle b, a c\rangle} \times \cdots \times E^{\left\langle b, a^{l-1} c\right\rangle} .
$$

Lemma 2.23. The group $\lim _{F \in \mathcal{F}}^{1} B(F)^{\times}$is uncountable.

Proof. According to statement b of Proposition 1.1, it suffices to show that the inverse system $\left(B(F)^{\times}\right)_{F \in \mathcal{F}}$ fails (ML). Examples 2.20 and 2.21 show that we shall need to consider nonabelian Galois groups and nonsplit extensions.

Fix an odd prime number $l$, and choose a prime number $p_{0}$ that splits completely in $\mathbb{Q}[\sqrt[l]{1}]$. Then $l \mid p_{0}-1$, and so there is a surjective homomorphism

$$
\left(\mathbb{Z} / p_{0} \mathbb{Z}\right)^{\times} \rightarrow C_{l} .
$$

Let $F_{0}$ be the subfield of $\mathbb{Q}\left[\sqrt[p_{0}]{1}\right]$ fixed by the kernel of one such homomorphism.

To prove that $\left(B(F)^{\times}\right)_{F \in \mathcal{F}}$ fails (ML) it suffices to show that, for each $L \in \mathcal{F}$ containing $F_{0}$, there exists an $E \in \mathcal{F}$ containing $F_{0}$ and such that

$$
\mathrm{Nm}_{B(E) / B\left(F_{0}\right)}\left(B(E)^{\times}\right) \not \supset \mathrm{Nm}_{B(L) / B\left(F_{0}\right)}\left(B(L)^{\times}\right) ;
$$

for then $E \cdot L \in \mathcal{F}$, but

$$
\mathrm{Nm}_{B(E \cdot L) / B\left(F_{0}\right)}\left(B(E \cdot L)^{\times}\right) \neq \mathrm{Nm}_{B(L) / B\left(F_{0}\right)}\left(B(L)^{\times}\right) .
$$

Choose a prime $p_{1}$ that splits completely in $L\left[\sqrt[l]{1}, \sqrt[l]{p_{0}}\right]$, and construct a cyclic extension $F_{1}$ of $\mathbb{Q}$ of degree $l$ by choosing a surjective homomorphism $\left(\mathbb{Z} / p_{1} \mathbb{Z}\right)^{\times} \rightarrow C_{l}$, as before. Then $F={ }_{\mathrm{df}} F_{0} \cdot F_{1}$ has Galois group

$$
\operatorname{Gal}(F / \mathbb{Q}) \cong C_{l} \times C_{l} .
$$

Let $G$ be as in Example 2.22, and consider the extension

$$
1 \rightarrow\langle b\rangle \rightarrow G \rightarrow\langle a, c\rangle \rightarrow 1 .
$$

Let

$$
\alpha: G /\langle b\rangle \rightarrow \operatorname{Gal}(F / \mathbb{Q})
$$

be the isomorphism sending $c$ to a generator of $\operatorname{Gal}\left(F_{0} / \mathbb{Q}\right)$ and $a$ to a generator of $\operatorname{Gal}\left(F_{1} / \mathbb{Q}\right)$. The only primes ramifying in $F$ are $p_{0}$ and $p_{1}$, and for $p=p_{0}$ or $p_{1}$ :

i) $l$ divides $p-1$ (because both primes split in $\mathbb{Q}[\sqrt[l]{1}]$ );

ii) for all primes $v$ of $F$ dividing $p, F_{v}$ is totally ramified over $\mathbb{Q}_{p}\left(p_{i}\right.$ is totally ramified in $F_{i}$; $p_{1}$ splits completely in $F_{0}$ by construction; $p_{0}$ splits completely in $F_{1}$ because it becomes an lth power in $\left.\mathbb{F}_{p_{1}}\right)$.

Now an argument of Scholz and Reichardt (see [Ser92, Theorem 2.1.3]) shows that there exists a Galois extension $E$ of $\mathbb{Q}$ containing $F$ for which there is a commutative diagram as follows.

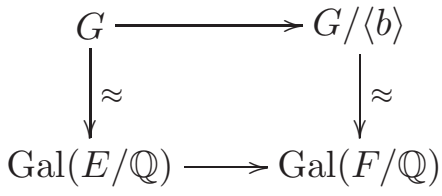




\section{J. S. Milne}

Note that $E$, being Galois of odd degree over $\mathbb{Q}$, must be totally real.

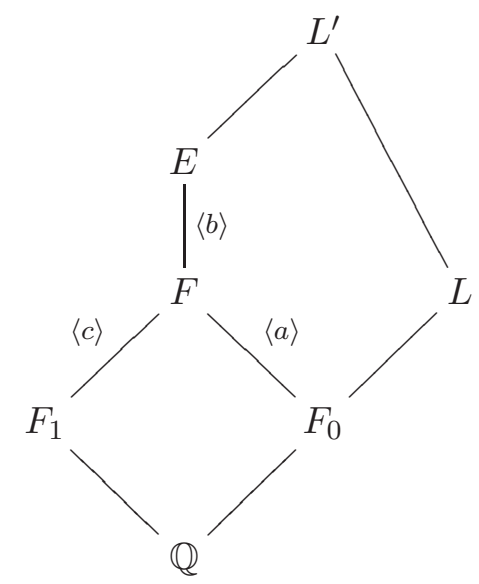

I claim that the image of $B(E)^{\times}$in $F_{0}^{\{c\} \times}$ does not contain the image of $B(L)^{\times}$. In order to show this, it suffices to show that the image of $\left(B(E) \otimes \mathbb{Q}_{p_{1}}\right)^{\times}$in $\left(F_{0}^{\{c\}} \otimes \mathbb{Q}_{p_{1}}\right)^{\times}=\mathbb{Q}_{p_{1}}^{\times}$does not contain the image of $\left(B(L) \otimes \mathbb{Q}_{p_{1}}\right)^{\times}$. But, because $p_{1}$ splits completely in $L$, the second image is $\mathbb{Q}_{p_{1}}^{\times}$. On the other hand, $p_{1}$ is totally ramified in each field $E^{\left\langle b, a^{j} c\right\rangle}$ (because $E^{\left\langle b, a^{j} c\right\rangle} \cdot F_{0}=F,\left(p_{1}\right)=\mathfrak{p}_{1}^{l} \cdots \mathfrak{p}_{l}^{l}$ in $F$, and $p_{1}$ splits completely in $F_{0}$ ), and so, for each $v \mid p, E_{v}^{\left\langle b, a^{j} c\right\rangle}$ is the (unique) tamely ramified cyclic extension of $\mathbb{Q}_{p_{1}}$ of degree $l$. Thus, the image of $\prod_{v} E_{v}^{\left\langle b, a^{j} c\right\rangle \times}$ in $\mathbb{Q}_{p_{1}}^{\times}$does not contain $\mathbb{Z}_{p_{1}}^{\times}$.

\section{Characterizing the period torsor}

Theorem 2.24. Let $K$ be a $C M$-subfield of $\mathbb{C}$, Galois over $\mathbb{Q}$.

a) If $[K: \mathbb{Q}]<\infty$ and $K$ contains a quadratic imaginary field, then $\mathrm{P}\left(G_{\mathrm{B}}^{\mathrm{CM}, K} \rightarrow \Gamma ; P^{\text {Art }}\right)$ contains exactly one isomorphism class, which is represented by $P^{\mathrm{CM}, K} \rightarrow P^{\mathrm{Art}}$.

b) If $K=\mathbb{Q}^{\mathrm{cm}}$, then $\mathrm{P}\left(G_{\mathrm{B}}^{\mathrm{CM}, K} \rightarrow \Gamma ; P^{\text {Art }}\right)$ contains uncountably many isomorphism classes.

Proof. According to Proposition 2.10, the isomorphism classes in $\mathrm{P}\left(G_{\mathrm{B}}^{\mathrm{CM}, K} \rightarrow \Gamma ; P^{\text {Art }}\right)$ are classified by $H^{1}\left(\mathbb{Q},{ }_{f} S^{K}\right)$. Therefore, statements a and b follow respectively from Propositions 2.15 and 2.11.

Remark 2.25. In fact, we have shown that the uncountable group $\varliminf_{K}^{1}{ }_{f} S^{K}(\mathbb{Q})$ acts simply transitively on the set of isomorphism classes of objects in $\mathrm{P}\left(G_{\mathrm{B}}^{\mathrm{CM}} \rightarrow \Gamma ; P^{\mathrm{Art}}\right)$. To make this explicit, let $K_{0}$ be a quadratic imaginary field, and let

$$
K_{0} \subset \cdots \subset K_{n} \subset \cdots \subset \mathbb{Q}^{\mathrm{cm}}
$$

be a sequence of CM-fields with union $\mathbb{Q}^{\mathrm{cm}}$. Let $S_{n}=S^{K_{n}}$ and $P_{n}^{\mathrm{CM}}=P^{\mathrm{CM}, K_{n}}$. From an element $s=\left(s_{n}\right)_{n \in \mathbb{N}}$ of $\prod_{f} S_{n}(\mathbb{Q})$ we obtain a $v$-morphism of torsors $P^{\mathrm{CM}}(s) \rightarrow P^{\text {Art }}$ by modifying the transition maps in $P^{\mathrm{CM}}$ : define

$$
P^{\mathrm{CM}}(s)=\lim _{n \in \mathbb{N}}\left(P_{n}^{\mathrm{CM}}, v_{n} \circ s_{n}\right)
$$

Then, the isomorphism class of $P^{\mathrm{CM}}(s) \rightarrow P^{\text {Art }}$ depends only on the class of $\left(s_{n}\right)$ in $\varliminf_{n}^{1} \bigsqcup_{n} S_{n}(\mathbb{Q})$, distinct classes in $\varliminf_{n}^{1}{ }_{f} S_{n}(\mathbb{Q})$ give nonisomorphic objects in $\mathrm{P}\left(G_{B}^{\mathrm{CM}} \rightarrow \Gamma ; P^{\mathrm{Art}}\right)$, and every object in $\mathrm{P}\left(G_{B}^{\mathrm{CM}} \rightarrow \Gamma ; P^{\mathrm{Art}}\right)$ is isomorphic to $P^{\mathrm{CM}}(s) \rightarrow P^{\text {Art }}$ for some $s \in \prod_{f} S_{n}(\mathbb{Q})$.

Remark 2.26. There remains the problem of characterizing the isomorphism class of $P^{\mathrm{CM}} \rightarrow P^{\text {Art }}$. One may hope that it is uniquely determined by its isomorphism classes in $\mathrm{P}\left(\left(G_{\mathrm{B}}^{\mathrm{CM}}\right)_{/ \mathbb{Q}_{l}} \rightarrow \Gamma_{/ \mathbb{Q}_{l}}, P_{/ \mathbb{Q}_{l}}^{\mathrm{Art}}\right)$ 


\section{PeRiods of ABELIAN VARIETIES}

for $l=2,3, \ldots, \infty$, i.e. that the kernel of

$$
H^{1}\left(\mathbb{Q}, f_{f} S\right) \rightarrow \prod_{l} H^{1}\left(\mathbb{Q}_{l}, f_{f} S\right)
$$

is zero, but the calculations I have made in this direction do not look promising. Note that ${ }_{f} S_{/ \mathbb{R}}$ is a countable product of copies of $\mathbb{G}_{m}$, and so $H^{1}\left(\mathbb{R},{ }_{f} S\right)=0$.

\section{Periods of abelian varieties}

For a class of abelian varieties $\mathcal{A}$ over $\mathbb{Q},\langle\mathcal{A}\rangle^{\otimes}$ denotes the category of motives based on the abelian varieties in $\mathcal{A}$, the projective spaces, and the zero-dimensional varieties. Let

$$
\begin{aligned}
G_{\mathrm{B}}^{\mathcal{A}} & =\mathcal{A} u t^{\otimes}\left(\omega_{\mathrm{B}} \mid\langle\mathcal{A}\rangle^{\otimes}\right), \\
G_{\mathrm{dR}}^{\mathcal{A}} & =\mathcal{A} u t^{\otimes}\left(\omega_{\mathrm{dR}} \mid\langle\mathcal{A}\rangle^{\otimes}\right), \\
P^{\mathcal{A}} & =\mathcal{I}_{s o m}{ }^{\otimes}\left(\omega_{\mathrm{B}}, \omega_{\mathrm{dR}}\right) .
\end{aligned}
$$

When $\mathcal{A}=\{A\}$, we write $A$ for $\mathcal{A}$.

3.1 The inclusion of the Artin motives into $\langle\mathcal{A}\rangle^{\otimes}$ defines a homomorphism

$$
G_{\mathrm{B}}^{\mathcal{A}} \rightarrow \Gamma
$$

This homomorphism is surjective, and its kernel is the identity component $\left(G_{\mathrm{B}}^{\mathcal{A}}\right)^{\circ}$ of $G_{\mathrm{B}}^{\mathcal{A}}$ (see $[\mathrm{DM} 82$, $6.23]) .{ }^{5}$ In particular, for a single abelian variety $A$, there is an exact sequence

$$
1 \rightarrow \mathrm{MT}(A) \rightarrow G_{\mathrm{B}}^{A} \rightarrow \Gamma \rightarrow 1 .
$$

3.2 For a single abelian variety $A$, there is a unique homomorphism

$$
\mathbb{S} \rightarrow \operatorname{MT}(A)_{/ \mathbb{R}}^{\mathrm{ab}}
$$

sending $h_{\text {can }}$ onto $\left(h^{A}\right)^{\text {ab }}$ where, as usual, $h^{A}: \mathbb{S} \rightarrow \mathrm{MT}(A)_{/ \mathbb{R}}$ is the homomorphism defining the Hodge structure on $H^{*}(A, \mathbb{Q})$. This homomorphism is surjective, and it factors through $S^{K}$ if and only if $K$ contains the reflex field of $\left(\operatorname{MT}(A), h^{A}\right)^{\mathrm{ab}}$.

3.3 On combining the last two statements, we see that if $A$ is an abelian variety over $\mathbb{Q}$ such that $S^{K} \rightarrow \mathrm{MT}(A)^{\text {ab }}$ is an isomorphism for some $K$, then

$$
1 \rightarrow \mathrm{MT}(A)^{\mathrm{der}} \rightarrow G_{\mathrm{B}}^{A} \rightarrow G_{\mathrm{B}}^{\mathrm{CM}, K} \rightarrow 1
$$

is exact.

\section{Some Hasse principles}

We first prove an elementary structure theorem.

Lemma 3.4. Every semisimple group $H$ over $\mathbb{Q}$ such that $H_{/ \mathbb{Q} \text { al }}$ is a product of simple groups is isomorphic to a product of groups $H_{i}$ of the form $H_{i}=\operatorname{Res}_{F_{i} / \mathbb{Q}} N_{i}$ with $F_{i}$ a number field and $N_{i}$ an absolutely simple group over $F_{i}$.

Proof. To give a semisimple group over $\mathbb{Q}$ is the same as to give a semisimple group $H$ over $\mathbb{Q}^{\text {al }}$ together with a descent datum $\left(\alpha_{\sigma}\right)_{\sigma \in \Gamma}$. Here $\alpha_{\sigma}$ is an isomorphism $\sigma H \rightarrow H, \alpha_{\sigma} \circ \sigma \alpha_{\tau}=\alpha_{\sigma \tau}$ for

\footnotetext{
${ }^{5}$ This applies because of Deligne's theorem [Del82a] that all Hodge classes on abelian varieties are absolutely Hodge.
} 
all $\sigma$ and $\tau$, and there is a continuity condition (namely, there exists a model $\left(H^{\prime}, \alpha: H_{/ \mathbb{Q}^{\text {al }}}^{\prime} \rightarrow H\right.$ ) of $H$ over some field $F$ of finite degree over $\mathbb{Q}$ such that $\alpha_{\sigma} \circ \sigma \alpha=\alpha$ for all $\sigma$ fixing $F$ ).

First consider a pair $\left(H,\left(\alpha_{\sigma}\right)_{\sigma \in \Gamma}\right)$ with $H$ an adjoint group. Write $H=\prod_{i \in I} H_{i}$ as a product of simple groups. For each $\sigma \in \Gamma$, there is a permutation (also denoted $\sigma$ ) of $I$ such that $\alpha_{\sigma}$ is a product of isomorphisms

$$
\alpha_{\sigma}(i): \sigma H_{i} \rightarrow H_{\sigma i} .
$$

Let $J$ be an orbit of $\Gamma$ in $I$, let $j \in J$, and let $\Gamma_{j}=\{\sigma \in \Gamma \mid \sigma j=j\}$. Then $\left(\alpha_{\sigma}(j)\right)_{\sigma \in \Gamma_{j}}$ is a descent datum on $H_{j}$, and $\left(\prod_{i \in J} \alpha_{\sigma}(i)\right)_{\sigma \in \Gamma}$ is a descent datum on $\prod_{i \in J} H_{i}$. The first defines a model $N_{j}$ of $H_{j}$ over $F_{j}={ }_{\mathrm{df}} \mathbb{Q}^{\Gamma_{j}}$, which is absolutely simple, and the second defines a model $M_{J}$ of $\prod_{i \in J} H_{i}$ over $\mathbb{Q}$, which is isomorphic to $\operatorname{Res}_{F_{j} / \mathbb{Q}} N_{j}$. Now $\prod_{J \in \Gamma \backslash I} M_{J}$ is a semisimple group over $\mathbb{Q}$ giving rise to $\left(H,\left(\alpha_{\sigma}\right)_{\sigma \in \Gamma}\right)$ over $\mathbb{Q}^{\text {al }}$.

Next consider a pair $\left(H,\left(\alpha_{\sigma}\right)_{\sigma \in \Gamma}\right)$ with $H$ a product $H=\prod_{i \in I} H_{i}$ of simple groups. Then $\left(\alpha_{\sigma}\right)_{\sigma}$ defines a descent datum $\left(\alpha_{\sigma}^{\text {ad }}\right)_{\sigma}$ on $H^{\text {ad }}$, and, as above, $\alpha_{\sigma}^{\text {ad }}$ is a product of isomorphisms $\alpha_{\sigma}^{\mathrm{ad}}(i): \sigma H_{i}^{\mathrm{ad}} \rightarrow H_{\sigma i}^{\mathrm{ad}}$. Consider the following diagram.

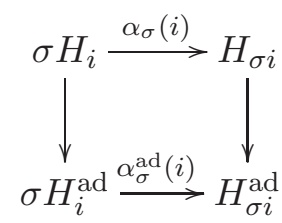

Here $\alpha_{\sigma}(i)$ is the composite

$$
\sigma H_{i} \hookrightarrow \sigma H \stackrel{\alpha_{\sigma}}{\longrightarrow} H \stackrel{\text { project }}{\longrightarrow} H_{i} .
$$

Because the diagram commutes, $\alpha_{\sigma}$ and $\prod_{i \in I} \alpha_{\sigma}(i)$ differ by a map from $\sigma H$ into the centre of $H$, which must be trivial because $\sigma H$ is connected. Thus, $\alpha_{\sigma}=\prod_{i \in I} \alpha_{\sigma}(i)$, and the same argument as in the preceding paragraph completes the proof of the lemma.

We define the index (of connectivity) of a semisimple algebraic group $H$ to be the degree of the universal covering $\tilde{H} \rightarrow H$. Thus, for an isogeny $a: H^{\prime} \rightarrow H$ of semisimple groups,

$$
\operatorname{index}\left(H^{\prime}\right) \leqslant \operatorname{index}(H) \text {, }
$$

with equality if and only if $a$ is an isomorphism.

Consider the following condition on a semisimple algebraic group $H$ over $\mathbb{Q}$ :

$$
H_{/ \mathbb{Q}^{\text {al }}} \text { is a product of simple groups of index } 1 \text { or } 2 .
$$

Proposition 3.5. For any semisimple group $H$ over $\mathbb{Q}$ satisfying (*), the map

$$
H^{1}(\mathbb{Q}, H) \rightarrow \prod_{l=2, \ldots, \infty} H^{1}\left(\mathbb{Q}_{l}, H\right)
$$

is injective.

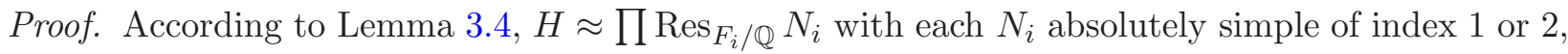
and

$$
H^{1}(\mathbb{Q}, H) \approx \prod_{i} H^{1}\left(\mathbb{Q}, H_{i}\right) \approx \prod_{i} H^{1}\left(F_{i}, N_{i}\right)
$$

Therefore, the proposition follows from the next two lemmas.

Lemma 3.6. For any simply connected semisimple group $H$ over a number field $F$, the map

is bijective.

$$
H^{1}(F, H) \rightarrow \prod_{v \text { real }} H^{1}\left(F_{v}, H\right)
$$




\section{PERIODS OF ABELIAN VARIETIES}

Proof. This is the theorem of Kneser, Harder, and Chernousov (see [PR94, Theorem 6.6, p. 286]).

Lemma 3.7. For any semisimple group $H$ of index 2 over a number field $F$,

$$
H^{1}(F, H) \rightarrow \prod_{v} H^{1}\left(F_{v}, H\right) \quad(v \text { runs over all primes of } F)
$$

is injective.

Proof. Platonov and Rapinchuk [PR94, Remark p. 337] note that the map has trivial kernel. The lemma can now be proved by a twisting argument, because any form of a semisimple group of index 2 again has index 2 .

Proposition 3.8. Let $H$ be a semisimple group over $\mathbb{Q}$ satisfying (*). If two cohomology classes in $H^{1}(\mathbb{Q}, \tilde{H})$ have the same image in $H^{1}(\mathbb{R}, H)$, then they have the same image in $H^{1}(\mathbb{Q}, H)$. In other words, $H^{1}(\mathbb{Q}, H) \rightarrow H^{1}(\mathbb{R}, H)$ is injective on the image of $H^{1}(\mathbb{Q}, \tilde{H}) \rightarrow H^{1}(\mathbb{Q}, H)$.

Proof. In the diagram

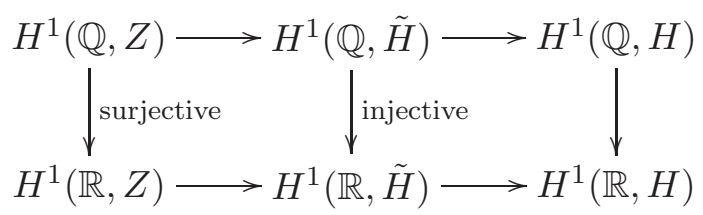

the rows are exact in the sense that the fibres of the second map are the orbits of the natural action of the first group on the middle set. The second vertical arrow is injective by Lemma 3.6. A diagram chase will complete the proof once we show that the first vertical arrow is surjective. In proving this, we may assume (Lemma 3.4) that $H=\operatorname{Res}_{F / \mathbb{Q}} N$ with $N$ an absolutely simple group of index 2 over a number field $F$. Then $Z=\operatorname{Res}_{F / \mathbb{Q}} \mu_{2}$, and so $H^{1}(\mathbb{Q}, Z) \rightarrow H^{1}(\mathbb{R}, Z)$ is the $\operatorname{map} F^{\times} / F^{\times 2} \rightarrow(F \otimes \mathbb{R})^{\times} /(F \otimes \mathbb{R})^{\times 2}$, which is surjective by the weak approximation theorem.

Remark 3.9. The proof of Proposition 3.8 shows that for any central extension

$$
1 \rightarrow Z \rightarrow G^{\prime} \rightarrow G \rightarrow 1
$$

of algebraic groups, the map $H^{1}(\mathbb{Q}, G) \rightarrow H^{1}(\mathbb{R}, G)$ is injective on the image of $H^{1}\left(\mathbb{Q}, G^{\prime}\right)$ in $H^{1}(\mathbb{Q}, G)$ provided

a) the $\operatorname{map} H^{1}(\mathbb{Q}, Z) \rightarrow H^{1}(\mathbb{R}, Z)$ is surjective;

b) the map $H^{1}\left(\mathbb{Q}, G^{\prime}\right) \rightarrow H^{1}\left(\mathbb{R}, G^{\prime}\right)$ is injective.

Proposition 3.10. Let $G$ be connected reductive group over $\mathbb{Q}$ such that $G^{\text {der }}$ is simply connected.

a) If $H^{1}\left(\mathbb{Q}, G^{\mathrm{ab}}\right) \rightarrow \prod_{l} H^{1}\left(\mathbb{Q}_{l}, G^{\mathrm{ab}}\right)$ is injective, then so also is $H^{1}(\mathbb{Q}, G) \rightarrow \prod_{l} H^{1}\left(\mathbb{Q}_{l}, G\right)$ (product over $l=2,3,5, \ldots, \infty)$.

b) If $H^{1}\left(\mathbb{Q}, G^{\mathrm{ab}}\right) \rightarrow H^{1}\left(\mathbb{R}, G^{\mathrm{ab}}\right)$ is injective, then so also is $H^{1}(\mathbb{Q}, G) \rightarrow H^{1}(\mathbb{R}, G)$.

Proof. a) See [Del71, 5.12].

(Because $G^{\text {der }}$ is simply connected, $H^{1}\left(\mathbb{Q}_{l}, G^{\text {der }}\right)=0$ for $l \neq \infty$ [PR94, Theorem 6.4, p. 284]. Using this, we obtain a commutative diagram with exact rows.

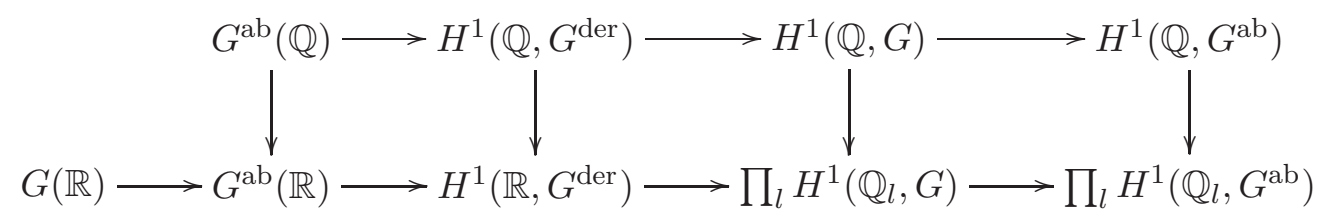




\section{J. S. MiLnE}

The image of $G(\mathbb{R})$ in $G^{\mathrm{ab}}(\mathbb{R})$ contains its identity component, and the real approximation theorem [San81, 3.5 (iii)] shows that $G^{\mathrm{ab}}(\mathbb{R})$ maps onto $\pi_{0}\left(G^{\mathrm{ab}}(\mathbb{R})\right)$. Now a diagram chase shows that any element of $H^{1}(\mathbb{Q}, G)$ that is locally zero is zero. To show that two elements $c$ and $c^{\prime}$ of $H^{1}(\mathbb{Q}, G)$ are equal when they are locally, choose a torsor representing $c$, and twist the groups by it; cf. [Ser64, I 5.3].)

b) The proof is similar, but easier.

\section{Characterizing $P^{\mathrm{AV}} \rightarrow P^{\mathrm{CM}}$}

Let $\mathrm{AV}(\mathbb{Q})=\langle\mathcal{A}\rangle^{\otimes}$ with $\mathcal{A}$ the class of all abelian varieties over $\mathbb{Q}$.

Theorem 3.11. Let $A$ be an abelian variety over $\mathbb{Q}$. If $\operatorname{MT}(A)^{\text {der }}$ satisfies $(*)$ and $d^{6} \mathrm{MT}(A)^{\mathrm{ab}} \cong S^{K}$ for some $K$, then the isomorphism class of $P^{A} \rightarrow P^{\mathrm{CM}, K}$ is uniquely determined by its classes over $\mathbb{Q}_{l}(l=2,3, \ldots, \infty)$.

Proof. According to Proposition 1.10, the isomorphism classes in $\mathrm{P}\left(G_{\mathrm{B}}^{A} \rightarrow G_{\mathrm{B}}^{\mathrm{CM}, K} ; P^{\mathrm{CM}, K}\right)$ are classified by $H^{1}(\mathbb{Q}, H)$ where $H$ is the twist of $\operatorname{Ker}\left(G_{\mathrm{B}}^{A} \rightarrow G_{\mathrm{B}}^{\mathrm{CM}, K}\right)$ by the period torsor. But according to $\S 3.3$, this kernel is $\operatorname{MT}(A)^{\text {der }}$, and so the theorem follows from Proposition 3.5.

The next result shows that the abelian varieties over $\mathbb{Q}$ satisfying the conditions of Theorem 3.11 are cofinal among all abelian varieties over $\mathbb{Q}$ for the relation $A \prec B$ if $h A$ is isomorphic to an object of $\langle B\rangle^{\otimes}$.

Proposition 3.12. Let $A$ be an abelian variety over $\mathbb{Q}$. For any sufficiently large $C M$-subfield $K$ of $\mathbb{C}$, there exists an abelian variety $B$ over $\mathbb{Q}$ such that $\mathrm{MT}(A \times B)^{\text {der }}$ satisfies $(*)$ and $\mathrm{MT}(A \times B)^{\mathrm{ab}} \cong$ $S^{K}$.

This will be proved in the next subsection.

TheOrem 3.13. The isomorphism class of $P^{\mathrm{AV}} \rightarrow P^{\mathrm{CM}}$ is uniquely determined by its classes over $\mathbb{Q}_{l}(l=2,3, \ldots, \infty)$.

Proof. According to Proposition 1.10, the isomorphism classes in $\mathrm{P}\left(G_{\mathrm{B}}^{\mathrm{AV}} \rightarrow G_{\mathrm{B}}^{\mathrm{CM}} ; P^{\mathrm{CM}}\right)$ are classified by $H^{1}(\mathbb{Q}, H)$ where $H$ is the twist of $H^{\prime}=\operatorname{Ker}\left(G_{\mathrm{B}}^{\mathrm{AV}} \rightarrow G_{\mathrm{B}}^{\mathrm{CM}}\right)$ by the period torsor. Proposition 3.12 implies that

$$
H^{\prime} \cong \lim _{\longleftarrow} \operatorname{MT}(A)^{\text {der }}
$$

where $A$ runs over the abelian varieties satisfying the hypothesis of Theorem 3.11. Therefore $H$ is a countable product of simple algebraic groups satisfying (*), and so we can apply Corollary 1.14 and Proposition 3.5.

\section{Proof of Proposition 3.12}

We shall say that an algebraic group $H$ over a field $k$ of characteristic zero is a special orthogonal group if there exists vector space $V$ over $k$ of dimension $n>8$ and a nondegenerate quadratic form $q$ on $V$ such that $H \approx \mathrm{SO}(V, q)$. Such an $H$ is connected and simple, and there are isogenies of degree 2

$$
\tilde{H} \rightarrow H \rightarrow H^{\text {ad }},
$$

with $\tilde{H}$ the simply connected covering group of $H$ (a spinor group). Let $Z=Z \tilde{H}$. When $n$ is even $Z\left(k^{\mathrm{al}}\right) \approx C_{2} \times C_{2}$.

${ }^{6}$ We do not really need to assume $\operatorname{MT}(A)^{\text {ab }} \cong S^{K} ;$ it only makes the statement a little more pleasant. 


\section{PERIODS OF ABELIAN VARIETIES}

3.14 Recall from [Del79a] the following statements.

a) Let $A$ be an abelian variety over $\mathbb{C}$. Each $\mathbb{Q}$-simple factor $H$ of $\operatorname{MT}(A)^{\text {ad }}$ is of type $A, B, C$, $D^{\mathbb{R}}$, or $D^{\mathbb{H}}$ (notations as in [Del79a, 2.3.8]).

b) Let $A$ be an abelian variety over $\mathbb{C}$, and let $G=\operatorname{MT}(A)$. The homomorphism $h: \mathbb{S} \rightarrow G_{/ \mathbb{R}}$ defined by the Hodge structure on $H^{*}(A, \mathbb{Q})$ satisfies the following conditions:

i) the Hodge structure on the Lie algebra $\mathfrak{g}$ of $G$ defined by $\operatorname{Ad} \circ h: \mathbb{S} \rightarrow \operatorname{GL}(\mathfrak{g})$ is of type $\{(1,-1),(0,0),(-1,1)\}$

ii) ad $h(i)$ is a Cartan involution on $G^{\text {ad }}$;

iii) $h$ generates $G$ (i.e. there is no proper closed subgroup $G^{\prime} \subset G$ such that $h(\mathbb{S}) \subset G_{/ \mathbb{R}}^{\prime}$ ).

c) Let $H$ be a simple adjoint group over $\mathbb{Q}$, and let $h: \mathbb{S} / \mathbb{G}_{m} \rightarrow H / \mathbb{R}$ be a homomorphism satisfying the conditions $\mathrm{i}$, ii and iii in statement $\mathrm{b}$.

i) If $H$ is of type $A, B, C$, or $D^{\mathbb{R}}$, there exists an abelian variety $A$ over $\mathbb{C}$ such that $\operatorname{MT}(A)^{\text {der }}$ is simply connected and $\left(\mathrm{MT}(A), h^{A}\right)^{\text {ad }} \approx(H, h)$ (apply [Del79a, 2.3.10]).

ii) Suppose $H$ is of type $D^{\mathbb{H}}$. Then $H=\operatorname{Res}_{F / \mathbb{Q}} N$ for some absolutely simple group $N$ over a totally real field $F$, and we let $H^{\prime}=\operatorname{Res}_{F / \mathbb{Q}} N^{\prime}$ where $N^{\prime} \rightarrow N$ is the double covering of $N$ that is an inner form of a special orthogonal group [Del79a, 2.3.8]. Any homomorphism $\tilde{H} \rightarrow \mathrm{MT}(A)$ sending $h$ to $\left(h^{A}\right)^{\text {ad }}$ factors through $H^{\prime}$ [Del79a, 1.3.10]. There exists an abelian variety $A$ over $\mathbb{C}$ with $\operatorname{MT}(A)^{\text {der }} \approx H^{\prime}$ and $\left(\operatorname{MT}(A), h^{A}\right)^{\text {ad }} \approx(H, h)$ (apply [Del79a, 2.3.10]).

We shall need the following condition on a semisimple group $H$ over $\mathbb{Q}$ :

$H$ is a product of simple groups; a simple factor of $H_{\mathbb{R}}$ has index 2 if it is of type $D^{\mathbb{H}}$ and index 1 otherwise.

Definition 3.15. An abelian variety defined over a subfield of $\mathbb{C}$ is maximal if $\operatorname{MT}(A)^{\text {der }}$ satisfies $(* *)$.

Lemma 3.16. A finite product of maximal abelian varieties is maximal.

Proof. Let $A=\prod_{j} A_{j}$ where each $A_{j}$ is maximal. The canonical map $\operatorname{MT}(A) \rightarrow \prod \operatorname{MT}\left(A_{j}\right)$ is injective and its composite with any projection $\prod \mathrm{MT}\left(A_{j}\right) \rightarrow \mathrm{MT}\left(A_{j}\right)$ is surjective [Del82a, $\S 3$. For a judicious choice of simple factors $H_{i}$ of $\prod \mathrm{MT}\left(A_{j}\right)^{\mathrm{der}}$, the homomorphism $\operatorname{MT}(A)^{\text {ad }} \rightarrow \prod H_{i}^{\text {ad }}$ will be an isomorphism. Consider

$$
\prod \tilde{H}_{i} \rightarrow \mathrm{MT}(A)^{\mathrm{der}} \rightarrow \prod H_{i}
$$

Then $\prod \tilde{H}_{i} \rightarrow \operatorname{MT}(A)^{\text {der }}$ factors through $\prod H_{i}^{\prime}$ where $H_{i}^{\prime}$ is as in statement c(ii) of $\S 3.14$ when $H_{i}$ is of type $D^{\mathbb{H}}$ and equals $\tilde{H}_{i}$ otherwise. Consider

$$
\prod H_{i}^{\prime} \rightarrow \operatorname{MT}(A)^{\text {der }} \rightarrow \prod H_{i} .
$$

As $\prod H_{i}^{\prime}$ and $\prod H_{i}$ have the same index, the composite is an isomorphism, and so $\operatorname{MT}(A)^{\text {der }} \rightarrow \prod H_{i}$ is an isomorphism.

Remark 3.17. Let $A$ and $B$ be abelian varieties such that $\mathrm{MT}(A \times B)^{\text {ad }} \cong \mathrm{MT}(B)^{\text {ad }}$. If $B$ is maximal, then so also is $A \times B$. This can be proved the same way as Lemma 3.16; one only has to observe that, because of the condition on the adjoint groups, the $H_{i}$ in the proof can be chosen to be factors of $\operatorname{MT}(B)^{\text {der }}$.

Lemma 3.18. For any abelian variety $A$ over $\mathbb{C}$, there exists an abelian variety $B$ over $\mathbb{C}$ such that $\left(\mathrm{MT}(B), h^{B}\right)^{\mathrm{ad}} \approx\left(\mathrm{MT}(A), h^{A}\right)^{\mathrm{ad}}$ and $B$ is maximal. 
Proof. According to $\S 3.14$, for each simple factor $(H, h)$ of $\left(\operatorname{MT}(A), h^{A}\right)^{\text {ad }}$, there exists an abelian variety $B(H)$ such that $\left(\mathrm{MT}(B(H)), h^{B(H)}\right)^{\text {ad }} \approx(H, h)$ and $\mathrm{MT}(B(H))^{\text {der }}$ is the covering $H^{\prime}$ of $H$ in condition ii in statement $\mathrm{c}$ of $\S 3.14$ if $H$ is of type $D^{\mathbb{H}}$ and is simply connected otherwise. Take $B=\prod B(H)$. Because each $B(H)$ is maximal, so is $B$ (by Lemma 3.16).

Recall that, when $A \prec B$, there is a canonical surjection $c_{A, B}: \operatorname{MT}(B) \rightarrow \operatorname{MT}(A)$ carrying $h^{B}$ to $h^{A}$.

Lemma 3.19. An abelian variety $A$ over $\mathbb{C}$ is maximal if and only if, for all $B$ such that $A \prec B$ and $c_{A, B}^{\text {ad }}: \operatorname{MT}(B)^{\text {ad }} \rightarrow \operatorname{MT}(A)^{\text {ad }}$ is an isomorphism, $c_{A, B}^{\text {der }}: \operatorname{MT}(B)^{\text {der }} \rightarrow \mathrm{MT}(A)^{\text {der }}$ is an isomorphism.

Proof. Let $B^{\prime}$ be as in Lemma 3.18, and let $B=A \times B^{\prime}$. Then $A \prec B$ and $\operatorname{MT}(B)^{\text {ad }} \rightarrow \operatorname{MT}(A)^{\text {ad }}$ is an isomorphism, but $\mathrm{MT}(B)^{\mathrm{der}} \rightarrow \mathrm{MT}(A)^{\text {der }}$ is an isomorphism only if $A$ is maximal. This proves the 'if'.

Let $A$ be maximal. Then it is clear from statement c of $\S 3.14$ that it satisfies the condition.

Lemma 3.20. Let $A$ be an abelian variety over $\mathbb{C}$, and let $\tau$ be an automorphism of $\mathbb{C}$. Then $A$ is maximal if and only if $\tau A$ is maximal.

Proof. Recall that $\operatorname{MT}(A)=\mathcal{A} u t^{\otimes}\left(\omega_{\mathrm{B}}\right)$ and $\operatorname{MT}(\tau A)=\mathcal{A} u t^{\otimes}\left(\omega_{\tau}\right)$ where $\omega_{\tau}$ is the fibre functor on $\mathrm{AV}(\mathbb{C})$ sending $h X$ to $H^{*}(\tau X, \mathbb{Q})$. Therefore, from the theory of Tannakian categories, $\operatorname{MT}(\tau A) \cong$ ${ }^{P} \operatorname{MT}(A)$ where $P=\mathcal{I}$ som $\left(\omega_{\mathrm{B}}, \omega_{\tau}\right)$.

If $A$ is not maximal, then there exists an abelian variety $B$ such that $A \prec B$ and $\operatorname{MT}(A)^{\text {ad }} \cong$ $\operatorname{MT}(B)^{\text {ad }}$ but $\operatorname{MT}(A)^{\text {der }} \neq \operatorname{MT}(B)^{\text {der }}$. Clearly $\tau B$ has the same properties relative to $\tau A$, which proves that $\tau A$ is not maximal.

Lemma 3.21. Let $A_{1}$ and $A_{2}$ be abelian varieties over $\mathbb{C}$ such that $\left(\mathrm{MT}\left(A_{1}\right), h^{A_{1}}\right)^{\text {ad }} \approx\left(\operatorname{MT}\left(A_{2}\right)\right.$, $\left.h^{A_{2}}\right)^{\mathrm{ad}}$. If $A_{1}$ can be defined over $\mathbb{Q}^{\text {al }}$, then so also can $A_{2}$.

For a proof, see [BB99, 3.3].

Lemma 3.22. For any $C M$-subfield $K$ of $\mathbb{C}$ of finite degree over $\mathbb{Q}$, there exists an abelian variety $A$ over $\mathbb{C}$ such that the canonical map $S^{K} \rightarrow \mathrm{MT}(A)$ is an isomorphism. If $K$ is Galois over $\mathbb{Q}$, then $A$ may be chosen to be defined over $\mathbb{Q}$.

Proof..$^{7}$ First recall that the CM-types on $K$ generate $X^{*}\left(S^{K}\right)$ : in fact, if $\psi=\tau_{1}+\cdots+\tau_{g}$ is one CM-type on $K$, then the CM-types $\psi_{i}=\tau_{i}+\sum_{j \neq i} \iota \tau_{j}(i=1, \ldots, g)$ and $\bar{\phi}=\sum \iota \tau_{j}$ form a basis for the $\mathbb{Z}$-module $X^{*}\left(S^{K}\right)$.

Let $B$ be a simple abelian variety over $\mathbb{C}$ of CM-type $(E, \phi)$. For each $\rho: E \rightarrow \mathbb{Q}^{\text {al }}$ and $\tau \in$ $\operatorname{Gal}\left(\mathbb{Q}^{\mathrm{cm}} / \mathbb{Q}\right)$, define $\psi_{\rho}(\tau)=\phi\left(\tau^{-1} \circ \rho\right)$. Then, as $\rho$ runs over the embeddings of $E$ into $\mathbb{Q}^{\text {al }}, \psi_{\rho}$ runs over a $\Gamma$-orbit of CM-types on $\mathbb{Q}^{\mathrm{cm}}$. The map $B \mapsto\left\{\psi_{\rho}\right\}$ defines a bijection from the set of isogeny classes of simple abelian varieties of CM-type over $\mathbb{Q}^{\text {al }}$ to the set of $\Gamma$-orbits of CM-types on $\mathbb{Q}^{\mathrm{cm}}$. A $\tau \in \Gamma$ fixes the reflex field $K$ of $B$ if and only if $\tau \phi=\phi$. Each $\psi_{\rho}$ is the extension to $\mathbb{Q}^{\mathrm{cm}}$ of a primitive CM-type on $K$, and the kernel of $S^{K} \rightarrow \mathrm{MT}(B)$ is the intersection of the kernels of the $\psi_{\rho}$ (see $[$ Mil99, $\S 2]$ ). For any automorphism $\sigma$ of $\mathbb{C}, \sigma B$ has reflex field $\sigma K$.

Choose a finite set of $B$ with reflex field contained in $K$ such that the corresponding $\psi$ generate $S^{K}$, and let $A$ be their product. The canonical map $S^{K} \rightarrow \mathrm{MT}(A)$ is then an isomorphism. According to $\S 2.7$, we may take $A$ to be defined over a subfield $k$ of $\mathbb{C}$ of finite degree over $\mathbb{Q}$. Then, if $K$ is Galois over $\mathbb{Q}, A_{*}={ }_{\mathrm{df}} \operatorname{Res}_{k / \mathbb{Q}} A$ has reflex field contained in $K$, and the canonical map $S^{K} \rightarrow \operatorname{MT}\left(A_{*}\right)$ is an isomorphism.

${ }^{7}$ For slightly weaker results, see [Mil90, I 4.6], [Wei94, 1.5.1] and [BB99, 3.5]. 


\section{PeRiods of ABELIAN VARIETIES}

Proof of Proposition 3.12. Let $A$ be an abelian variety over $\mathbb{Q}$. Choose $B$ as in Lemma 3.18. After Lemma 3.21, we may assume $B$ is defined over a number field $F$. Then $B_{*}=\prod_{\sigma \in \Sigma_{F / \mathbb{Q}}} \sigma B$ is defined over $\mathbb{Q}$. Let $K$ be any CM-subfield of $\mathbb{Q}^{\text {al }}$ containing the reflex field of $\left(\mathrm{MT}\left(A \times B_{*}\right), h^{A \times B_{*}}\right)^{\mathrm{ab}}$ and finite and Galois over $\mathbb{Q}$, and choose $C$ as in Lemma 3.22. Consider the abelian variety $A \times B_{*} \times C$. It is defined over $\mathbb{Q}$, and Lemmas 3.20 and 3.16 and Remark 3.17 show that it is maximal. By assumption, the reflex field of $\left(\mathrm{MT}\left(A \times B_{*} \times C\right), h^{A \times B_{*} \times C}\right)$ ab is contained in $K$, and so the canonical homomorphism $S \rightarrow \mathrm{MT}\left(A \times B_{*} \times C\right)^{\text {ab }}$ factors through $S^{K}$. But $\mathrm{MT}\left(A \times B_{*} \times C\right)$ surjects onto $\mathrm{MT}(C) \cong S^{K}$, and so $S^{K} \cong \mathrm{MT}\left(A \times B_{*} \times C\right)^{\mathrm{ab}}$. This completes the proof of Proposition 3.12 .

\section{Characterizing $\boldsymbol{P}^{\mathcal{H}} \rightarrow \boldsymbol{P}^{\mathrm{CM}}$}

Theorem 3.23. Let $A$ be an abelian variety over $\mathbb{Q}$. If $\operatorname{MT}(A)^{\text {der }}$ is simply connected and $\operatorname{MT}(A)^{\mathrm{ab}} \cong S^{K}$ for some $K$, then the isomorphism class of $P^{A} \rightarrow P^{\mathrm{CM}, K}$ is uniquely determined by its class over $\mathbb{R}$.

Proof. According to Proposition 1.10 and $\S 3.3$, the isomorphism classes in $\mathrm{P}\left(G_{\mathrm{B}}^{A} \rightarrow G_{\mathrm{B}}^{\mathrm{CM}, K} ; P^{\mathrm{CM}, K}\right)$ are classified by $H^{1}(\mathbb{Q}, H)$, where $H$ is the twist of $\operatorname{MT}(A)^{\text {der }}$ by the period torsor. Therefore $H$ is simply connected, and so this follows from the theorem of Kneser, Harder, and Chernousov (see Lemma 3.6).

Let $\mathcal{H}$ denote the class of abelian varieties $A$ over $\mathbb{Q}$ such that $\operatorname{MT}(A)^{\text {ad }}$ has no factor of type $D^{\mathbb{H}}$. Note that, because of condition ii in statement c of Proposition 3.14, any abelian variety for which $\operatorname{MT}(A)^{\text {der }}$ is simply connected lies in $\mathcal{H}$. The next result shows that the abelian varieties satisfying the conditions of Theorem 3.23 are cofinal among all abelian varieties in $\mathcal{H}$.

Proposition 3.24 [BB99, 3.2]. Let $A$ be an abelian variety in $\mathcal{H}$. For any sufficiently large $C M$-field $K$, there exists an abelian variety $B$ over $\mathbb{Q}$ such that $\operatorname{MT}(A \times B)^{\mathrm{ab}} \cong S^{K}$ and $\operatorname{MT}(A \times B)^{\text {der }}$ is simply connected.

Proof. When $A$ is in $\mathcal{H}$, the proof of Proposition 3.12 can be modified to show that $B$ can be chosen in such a way that $\operatorname{MT}(A \times B)^{\text {der }}$ is simply connected; in fact, this significantly simplifies the proof.

TheOREM 3.25. The isomorphism class of $P^{\mathcal{H}} \rightarrow P^{\mathrm{CM}}$ is uniquely determined by its isomorphism class over $\mathbb{R}$.

The proof is similar to that of Theorem 3.13.

Remark 3.26. The main theorem of [BB99, Theorem 1.5] states that the isomorphism class of $P^{\mathcal{H}} \rightarrow P^{\mathrm{CM}}$ is determined by the cohomology class of $P^{\mathcal{H}}$ in $\varliminf_{A \in \mathcal{H}} H^{1}\left(\mathbb{R}, G_{\mathrm{B}}^{A}\right)$. Theorem 3.25 states that the isomorphism class of $P^{\mathcal{H}} \rightarrow P^{\mathrm{CM}}$ is determined by its class in $H^{1}\left(\mathbb{R},\left(G_{\mathrm{dR}}^{\mathcal{H}}\right)^{\text {oder }}\right)$. There are maps

$$
H^{1}\left(\mathbb{R},\left(G_{\mathrm{dR}}^{\mathcal{H}}\right)^{\text {oder }}\right) \rightarrow \lim _{\overleftarrow{A \in \mathcal{H}}} H^{1}\left(\mathbb{R},\left(G_{\mathrm{dR}}^{A}\right)^{\text {oder }}\right) \rightarrow \lim _{\overleftarrow{A \in \mathcal{H}}} H^{1}\left(\mathbb{R},\left(G_{\mathrm{dR}}^{A}\right)^{\circ}\right) \rightarrow \lim _{\overleftarrow{A \in \mathcal{H}}} H^{1}\left(\mathbb{R}, G_{\mathrm{dR}}^{A}\right)
$$

The first is an isomorphism (by Corollary 1.14) and the third is injective [BB99, 4.4]. If the second were injective, their theorem would follow from Theorem 3.25, but I see no reason to expect this (the kernel of the second map is a quotient of ${ }_{f} S(\mathbb{R})$, which is a countable product of copies of $\mathbb{R}^{\times}$).

\section{Characterizing $P^{\mathrm{AV}} \rightarrow P^{\mathrm{CM}}$ in terms of a lifting property}

Let $A$ be an abelian variety over $\mathbb{Q}$ such that $\mathrm{MT}(A) \cong S^{K}$ for some CM-field $K$. Recall that $\operatorname{MT}(A)^{\text {der }}=\operatorname{Ker}\left(G^{A} \rightarrow G_{\mathrm{B}}^{\mathrm{CM}, K}\right)$. We say that $P^{A} \rightarrow P^{\mathrm{CM}, K}$ has the lifting property if there exists 


\section{J. S. MiLnE}

a surjective homomorphism $\tilde{G} \rightarrow G_{\mathrm{B}}^{A}$ such that the kernel of $\tilde{G} \rightarrow G_{\mathrm{B}}^{\mathrm{CM}, K}$ is the universal covering group of $\operatorname{MT}(A)^{\text {der }}$ and $P^{A}$ lifts to $\tilde{G}$.

Theorem 3.27. Let $A$ be an abelian variety over $\mathbb{Q}$ such that $\operatorname{MT}(A)^{\text {der }}$ satisfies $(*)$ and $S^{K} \cong$ $\operatorname{MT}(A)^{\text {ab }}$ for some $C M$-subfield $K$ of $\mathbb{C}$. Up to isomorphism, there exists at most one object $P \rightarrow$ $P^{\mathrm{CM}, K}$ in $\mathrm{P}\left(G^{A} \rightarrow G_{\mathrm{B}}^{\mathrm{CM}, K} ; P^{\mathrm{CM}, K}\right)$ such that

a) $P \rightarrow P^{\mathrm{CM}, K}$ has the lifting property, and

b) $\left(P \rightarrow P^{\mathrm{CM}, K}\right)_{\mathbb{R}} \approx\left(P^{A} \rightarrow P^{\mathrm{CM}, K}\right)_{\mathbb{R}}$.

Proof. Apply Proposition 3.8 to $H=\operatorname{MT}(A)^{\text {der }}$.

The notion of a lifting property extends in an obvious fashion to infinite sets of abelian varieties.

TheOREM 3.28. Up to isomorphism, there exists at most one object $P \rightarrow P^{\mathrm{CM}}$ in $\mathrm{P}\left(G_{\mathrm{B}}^{\mathrm{AV}} \rightarrow\right.$ $\left.G_{\mathrm{B}}^{\mathrm{CM}} ; P^{\mathrm{CM}}\right)$ such that

a) $P \rightarrow P^{\mathrm{CM}}$ has the lifting property, and

b) $\left(P \rightarrow P^{\mathrm{CM}}\right)_{\mathbb{R}} \approx\left(P^{\mathrm{AV}} \rightarrow P^{\mathrm{CM}}\right)_{\mathbb{R}}$.

The proof is similar to the proof of Theorem 3.13.

Remark 3.29. Deligne's hope that all Shimura varieties with rational weight are moduli varieties for motives [Del79a, p. 248] implies that $P^{\mathrm{AV}} \rightarrow P^{\mathrm{CM}}$ has the lifting property over $\mathbb{C}$. It would be interesting to prove this unconditionally.

\section{Characterizing $P^{\mathrm{AV}} \rightarrow P^{\mathrm{Art}}$}

TheOREm 3.30. Let $A$ be an abelian variety over $\mathbb{Q}$ such that $\operatorname{MT}(A)^{\text {ab }} \cong S^{K}$ for some $C M$-subfield $K$ of $\mathbb{C}$.

a) If $\operatorname{MT}(A)^{\text {der }}$ is simply connected, then the isomorphism class of $P^{A} \rightarrow P^{\text {Art }}$ is uniquely determined by its class over $\mathbb{R}$.

b) If $\operatorname{MT}(A)^{\text {der }}$ satisfies (*), then, up to isomorphism, there exists at most one object $P \rightarrow P^{\text {Art }}$ in $\mathrm{P}\left(G_{\mathrm{B}}^{A} \rightarrow \Gamma ; P^{\text {Art }}\right)$ having the lifting property and isomorphic to $P^{A} \rightarrow P^{\text {Art }}$ over $\mathbb{R}$.

Proof. a) According to Proposition 1.10, the isomorphism classes in $\mathrm{P}\left(G_{\mathrm{B}}^{A} \rightarrow \Gamma ; P^{\text {Art }}\right)$ are classified by $H^{1}(\mathbb{Q}, G)$, where $G$ is the twist of

$$
\operatorname{MT}(A) \stackrel{3.1}{=} \operatorname{Ker}\left(G_{\mathrm{B}}^{A} \rightarrow \Gamma\right)
$$

by the period torsor. Then $G^{\mathrm{ab}}={ }_{f} S^{K}$, and so $G$ satisfies the hypotheses of part b of Proposition 3.10 .

b) In this case, $G$ satisfies the hypotheses of Remark 3.9 .

Remark 3.31. For any abelian variety $A$ over $\mathbb{Q}$, the map $G_{\mathrm{dR}}^{A}(\mathbb{R}) \rightarrow{ }_{f} \Gamma(\mathbb{R})$ is surjective $[$ BB99, 4.4] and so

$$
H^{1}\left(\mathbb{R},\left(G_{\mathrm{dR}}^{A}\right)^{\circ}\right) \rightarrow H^{1}\left(\mathbb{R}, G_{\mathrm{dR}}^{A}\right)
$$

is injective. Therefore, for $P \rightarrow P^{\text {Art }}$ in $\mathrm{P}\left(G_{\mathrm{B}}^{A} \rightarrow \Gamma ; P^{\text {Art }}\right)$, the isomorphism class of $P \rightarrow P^{\text {Art }}$ over $\mathbb{R}$ is determined by the isomorphism class of $P$ over $\mathbb{R}$. 


\section{PERIODS OF ABELIAN VARIETIES}

\section{THEOREM 3.32 .}

a) There are uncountably many isomorphism classes in $\mathrm{P}\left(G_{\mathrm{B}}^{\mathcal{H}} \rightarrow \Gamma ; P^{\text {Art }}\right)$ that become isomorphic to $P^{\mathcal{H}} \rightarrow P^{\text {Art }}$ over $\mathbb{R}$.

b) If there exists one object $P \rightarrow P^{\text {Art }}$ in $\mathrm{P}\left(G_{\mathrm{B}}^{\mathrm{AV}} \rightarrow \Gamma ; P^{\text {Art }}\right)$ having the lifting property and isomorphic to $P^{\mathrm{AV}} \rightarrow P^{\mathrm{Art}}$ over $\mathbb{R}$, then there are uncountably many.

Proof. a) According to Proposition 1.10, the isomorphism classes in $\mathrm{P}\left(G_{\mathrm{B}}^{\mathcal{H}} \rightarrow \Gamma\right.$; $\left.P^{\text {Art }}\right)$ are classified by $H^{1}\left(\mathbb{Q},\left(G_{\mathrm{dR}}^{\mathcal{H}}\right)^{\circ}\right)$, where $\left(G_{\mathrm{dR}}^{\mathcal{H}}\right)^{\circ}$ is the twist of

$$
\left(G_{\mathrm{B}}^{\mathcal{H}}\right)^{\circ} \stackrel{3.1}{=} \operatorname{Ker}\left(G_{\mathrm{B}}^{\mathcal{H}} \rightarrow \Gamma\right)
$$

by the period torsor. For an $A \in \mathcal{H}$ with $\operatorname{MT}(A)^{\mathrm{ab}} \cong S^{K}$, consider

$$
0 \rightarrow\left(G_{\mathrm{dR}}^{A}\right)^{\text {oder }}(\mathbb{Q}) \rightarrow\left(G_{\mathrm{dR}}^{A}\right)^{\circ}(\mathbb{Q}) \rightarrow{ }_{f} S^{K}(\mathbb{Q}) \rightarrow H^{1}\left(\mathbb{Q},\left(G_{\mathrm{dR}}^{A}\right)^{\text {oder }}\right) .
$$

Because $\left(G_{\mathrm{dR}}^{\mathcal{H}}\right)^{\text {oder }}$ is a countable product of algebraic groups,

$$
\lim ^{1}\left(G_{\mathrm{dR}}^{A}\right)^{\text {oder }}(\mathbb{Q})=0=\lim ^{1} H^{1}\left(\mathbb{Q},\left(G_{\mathrm{dR}}^{A}\right)^{\text {oder }}\right)
$$

(by Corollary 1.14). Therefore,

$$
\lim ^{1}\left(G_{\mathrm{dR}}^{A}\right)^{\circ}(\mathbb{Q}) \cong \lim _{f}^{1} S^{K}(\mathbb{Q}),
$$

which is uncountable (by Proposition 2.18). Similarly,

$$
\lim ^{1}\left(G_{\mathrm{dR}}^{A}\right)^{\circ}(\mathbb{R}) \cong \lim _{f}^{1} S^{K}(\mathbb{R}),
$$

which is zero because ${ }_{f} S_{/ \mathbb{R}}^{K}$ is a product of copies of $\mathbb{G}_{m}$. In view of Proposition 1.12, this completes the proof.

b) This is similar to that of part a.

Remark 3.33. I expect that there are uncountably many distinct isomorphism classes in $\mathrm{P}\left(G_{\mathrm{B}}^{\mathrm{AV}} \rightarrow\right.$ $\left.\Gamma ; P^{\text {Art }}\right)$ that become equal to the class of $P^{\mathrm{AV}} \rightarrow P^{\text {Art }}$ over every field $\mathbb{Q}_{l}$.

\section{Characterizing $\boldsymbol{P}^{\mathrm{AV}}$}

Theorem 3.34. Let $A$ be an abelian variety over $\mathbb{Q}$ such that $\operatorname{MT}(A)^{\mathrm{ab}} \cong S^{K}$ for some $C M$-field $K$.

a) If $\operatorname{MT}(A)^{\mathrm{der}}$ is simply connected, then, up to isomorphism, $P^{A}$ is the only $G_{\mathrm{B}}^{A}$-torsor $P$ such that

i) $P \wedge \wedge_{\mathrm{B}}^{A} \Gamma \approx P^{\text {Art }}$, and

ii) $P_{/ \mathbb{R}} \approx P_{/ \mathbb{R}}^{A}$.

b) If $\operatorname{MT}(A)^{\text {der }}$ satisfies (*), then, up to isomorphism, there exists at most one $G_{\mathrm{B}}^{A}$-torsor $P$ such that
i) $P \wedge G_{\mathrm{B}}^{A} \Gamma \approx P^{\text {Art }}$,
ii) there exists a surjective homomorphism $u: G^{\prime} \rightarrow G_{\mathrm{B}}^{A}$ such that $u^{\mathrm{der}}: G^{\prime \mathrm{der}} \rightarrow\left(G_{\mathrm{B}}^{A}\right)^{\mathrm{der}}$ is the universal covering group of $\left(G_{\mathrm{B}}^{A}\right)^{\mathrm{der}}$ and $P=u P^{\prime}$ for some $G^{\prime}$-torsor, and
iii) $P_{/ \mathbb{R}} \approx P_{/ \mathbb{R}}^{A}$.

Proof. a) Let $P$ satisfy the conditions i and ii, and choose a morphism $P \rightarrow P^{\text {Art }}$. Then Theorem 3.30 and Remark 3.31 show that $\left(P \rightarrow P^{\text {Art }}\right) \approx\left(P^{A} \rightarrow P^{\text {Art }}\right)$.

b) This is similar to that of part a. 


\section{J. S. Milne}

\section{Other fields}

Hasse principles are known to hold for some fields other than number fields. For example, [Sch96] proves a Hasse principle for the Galois cohomology groups of connected linear algebraic groups over perfect fields with virtual cohomological dimension $\leqslant 1$. However, if the field $k$ is not countable, the affine group scheme $G$ attached to the category of abelian motives over $k$ will not be a countable inverse limit of algebraic groups. In particular, the relation of the flat cohomology group of $G$ to the Galois cohomology groups of its algebraic quotients is unknown, and so (pace [Fli01]) such results do not imply Hasse principles for period torsors.

\section{REFERENCES}

Ati61 M. F. Atiyah, Characters and cohomology of finite groups, Publ. Math. Inst. Hautes Études Sci. 9 (1961), 23-64.

BB99 D. Blasius and M. Borovoi, On period torsors, in Automorphic forms, automorphic representations, and arithmetic (Fort Worth, TX, 1996), Proc. Sympos. Pure Math., vol. 66, Part 1 (Amer. Math. Soc., Providence, RI, 1999), 1-8.

BD68 I. Bucur and A. Deleanu, with the collaboration of P. J. Hilton and N. Popescu, Introduction to the theory of categories and functors, Pure and Applied Mathematics, vol. XIX (Wiley Interscience, New York, 1968).

BK72 A. K. Bousfield and D. M. Kan, Homotopy limits, completions and localizations, Lecture Notes in Mathematics, vol. 304 (Springer, Berlin, 1972).

Bre90 L. Breen, Bitorseurs et cohomologie non abélienne, in The Grothendieck Festschrift, vol. I, Progr. Math., vol. 86 (Birkhäuser, Boston, MA, 1990), 401-476.

Del71 P. Deligne, Travaux de Shimura, Séminaire Bourbaki, 23ème année (1970/71), exp. no. 389 (1971) (Preliminary version).

Del79a P. Deligne, Variétés de Shimura: interprétation modulaire, et techniques de construction de modèles canoniques, in Automorphic forms, representations and L-functions (Oregon State Univ., Corvallis, OR, 1977), Part 2, Proc. Sympos. Pure Math., vol. XXXIII (Amer. Math. Soc., Providence, RI, 1979), 247-289.

Del79b P. Deligne, with an appendix by N. Koblitz and A. Ogus, Valeurs de fonctions L et périodes d'intégrales, in Automorphic forms, representations and L-functions (Oregon State Univ., Corvallis, OR, 1977), Part 2, Proc. Sympos. Pure Math., vol. XXXIII (Amer. Math. Soc., Providence, RI, 1979), 313-346.

Del82a P. Deligne, notes by J. Milne, Hodge cycles on abelian varieties, in Hodge cycles, motives, and Shimura varieties, Lecture Notes in Mathematics, vol. 900 (Springer, Berlin, 1982), 9-100.

Del82b P. Deligne, Motifs et groupe de Taniyama, in Hodge cycles, motives, and Shimura varieties, Lecture Notes in Mathematics, vol. 900 (Springer, Berlin, 1982), 261-279.

DM82 P. Deligne and J. S. Milne, Tannakian categories, in Hodge cycles, motives, and Shimura varieties, Lecture Notes in Mathematics, vol. 900 (Springer, Berlin, 1982), 101-228.

Fli01 Y. Z. Flicker, Motivic torsors, Israel J. Math. 122 (2001), 61-77.

Gir71 J. Giraud, Cohomologie non abélienne, Die Grundlehren der mathematischen Wissenschaften, Band 179 (Springer, Berlin, 1971).

Gra66 B. I. Gray, Spaces of the same n-type, for all n, Topology 5 (1966), 241-243.

Mil88 J. S. Milne, Automorphic vector bundles on connected Shimura varieties, Invent. Math. 92 (1988), 91-128.

Mil90 J. S. Milne, Canonical models of (mixed) Shimura varieties and automorphic vector bundles, in Automorphic forms, Shimura varieties, and L-functions, vol. I (Ann Arbor, MI, 1988), Perspect. Math., vol. 10 (Academic Press, Boston, MA, 1990), 283-414.

Mil99 J. S. Milne, Lefschetz motives and the Tate conjecture, Compositio Math. 117 (1999), 45-76. 


\section{PERIODS OF ABELIAN VARIETIES}

PR94 V. Platonov and A. Rapinchuk, Algebraic groups and number theory, translated from the 1991 Russian original by Rachel Rowen, Pure and Applied Mathematics, vol. 139 (Academic Press, Boston, MA, 1994).

San81 J.-J. Sansuc, Groupe de Brauer et arithmétique des groupes algébriques linéaires sur un corps de nombres, J. reine angew. Math. 327 (1981), 12-80.

Sch96 C. Scheiderer, Hasse principles and approximation theorems for homogeneous spaces over fields of virtual cohomological dimension one, Invent. Math. 125 (1996), 307-365.

Ser64 J.-P. Serre, Cohomologie galoisienne, Cours au Collège de France, 1962-1963, seconde édition, Lecture Notes in Mathematics, vol. 5 (Springer, Berlin, 1964).

Ser92 J.-P. Serre, Topics in Galois theory. Lecture notes prepared by Henri Damon [Henri Darmon], with a foreword by Darmon and the author, Research Notes in Mathematics, vol. 1 (Jones and Bartlett, Boston, MA, 1992).

Ser94 J.-P. Serre, Propriétés conjecturales des groupes de Galois motiviques et des représentations l-adiques, in Motives (Seattle, WA, 1991), Proc. Sympos. Pure Math., vol. 55, Part 1 (Amer. Math. Soc., Providence, RI, 1994), 377-400.

Tat76 J. Tate, Relations between $K_{2}$ and Galois cohomology, Invent. Math. 36 (1976), 257-274.

Wei94 W. Wei, Moduli fields of CM-motives applied to Hilbert's 12th problem, Preprint (1994), http://www.mathematik.uni-bielefeld.de/sfb343/preprints/pr94070.ps.gz.

Win90 J.-P. Wintenberger, Torseurs pour les motifs et pour les représentations p-adiques potentiellement de type CM, Math. Ann. 288 (1990), 1-8.

J. S. Milne math@jmilne.org

2679 Bedford Rd, Ann Arbor, MI 48104, USA 